\title{
On the flow past a circular cylinder from critical to super-critical Reynolds numbers: Wake topology and vortex shedding
}

\author{
I. Rodríguez ${ }^{\mathrm{a}}$, O. Lehmkuhl ${ }^{\mathrm{a}, \mathrm{b}}$, J. Chiva ${ }^{\mathrm{a}}$, R. Borrell ${ }^{\mathrm{b}}$, A. Oliva ${ }^{\mathrm{a}, *}$ \\ ${ }^{a}$ Heat and Mass Transfer Technological Centre (CTTC), Universitat Politècnica de \\ Catalunya-BarcelonaTech, ETSEIAT, Colom 11, 08222 Terrassa (Barcelona), Spain. \\ ${ }^{b}$ Termo Fluids, S.L., Avda. Jaquard, 97 1-E, 08222 Terrassa (Barcelona), Spain
}

\section{Abstract}

Large-eddy simulations (LES) of the flow past a circular cylinder are used to investigate the flow topology and the vortex shedding process at Reynolds numbers $R e=$ $2.5 \times 10^{5}-8.5 \times 10^{5}$. This range encompasses both the critical and super-critical regimes. As the flow enters the critical regime, major changes occur which affect the flow configuration. Asymmetries in the flow are found in the critical regime, whereas the wake recovers its symmetry and stabilises in the super-critical regime. Wake characteristic lengths are measured and compared between the different Reynolds numbers. It is shown that the super-critical regime is characterised by a plateau in the drag coefficient at about $C_{D} \approx 0.22$, and a quasi-stable wake which has a non-dimensional width of $d_{w} / D \approx 0.4$. The periodic nature of the flow is analysed by means of measurements of the unsteady drag and lift coefficients. Power spectra of the lift fluctuations are computed. Wake vortex shedding is found to occur for both regimes investigated, although a jump in frequencies is observed when the flow enters the super-critical regime. In this regime, non-dimensional vortex-shedding frequency is almost constant and equal to $S t=f_{v s} D / U_{r e f} \approx 0.44$. The analysis also shows a steep decrease in the fluctuating lift when entering the super-critical regime. The combined analysis of both wake topology and vortex shedding complements the physical picture of a stable and highly coherent flow in the super-critical regime.

Keywords: LES, critical and super-critical Reynolds numbers, vortex shedding, coherent

\footnotetext{
*Tel: +34 93739 8192; fax: +3493739 8101

Email address: cttc@cttc.upc.edu ( A. Oliva) 
flow, wake topology

\section{Introduction}

It is well known that the wake topology in the flow past a circular cylinder remains almost unchanged up to Reynolds number $\sim 10^{5}$ (Williamson, 1996). Then, at $R e \sim 2 \times 10^{5}$ major changes take place entailing flow separation, turbulence transition in the detached shear layers, reattachment of the flow and turbulent separation of the boundary layer on the rearward of the cylinder. Furthermore, a dramatic decrease in the drag coefficient occurs between Reynolds numbers $2 \times 10^{5}$ and $5 \times 10^{5}$. In this regime, a laminar separation bubble (LSB) on one side of the cylinder is formed as a consequence of the detachment and further reattachment of the boundary layer (Bearman, 1965; Schewe, 1983; Lehmkuhl et al., 2014). This asymmetry in the flow causes asymmetric forces on the cylinder, the lift coefficient being greater than zero. The formation of a second LSB on the other side of the cylinder and the recovery of the symmetry of the flow marks the onset of the super-critical regime. By entering the super-critical regime, the drag coefficient remains almost constant in the whole range being about $C_{D} \approx 0.2$ (Delany and Sorensen, 1953; Achenbach, 1968; Shih et al., 1993), whereas high values of the non-dimensional vortex shedding (Strouhal number $S t=$ $f_{v s} D / U_{r e f}$, where $f_{v s}$ is the vortex shedding frequency, $U_{r e f}$ is the free-stream velocity and $D$ the cylinder diameter) have been reported (see for instance Achenbach and Heinecke, 1981; Schewe, 1983).

Thorough observations and measurements of the wake behind cylinders have been made mainly for the laminar and sub-critical regimes (e.g. Roshko, 1954; Bloor and Gerrard, 1966; Cantwell and Coles, 1983), but less work has been carried out in the critical and super-critical regimes, where most of the studies have been focused on measuring loads on the cylinder surface and vortex shedding (Delany and Sorensen, 1953; Achenbach, 1968; Shih et al., 1993). Wieselsberger (1922) was one of the first researchers to point out the narrowing of the wake beyond the critical regime and the shifting of the separation point towards the rear of the cylinder. Spitzer (1965) performed experiments in the super-critical regime and measured forces on the cylinder surface. He also carried out measurements of 
the stream-wise velocity and its fluctuations in the wake, giving an idea of the wake width and its variations in this regime. Roshko (1961), although did not carry out direct measurements of the wake, described the changes occurring, especially those relative to the wake narrowing.

Regarding the vortex shedding, contrary to the sub-critical regime where consistent measurements of this parameter have been carried out, there is little information in the range of critical to super-critical Reynolds numbers, the values reported being quite scattered. The pioneering work of Relf and Simmons (1924) accounts for vortex shedding measurements in a large range of Reynolds numbers, including the critical and super-critical regimes up to $R e=10^{6}$. However, at these high Reynolds numbers their results were scattered and, even though they measured an increase in the Strouhal number, they concluded that the flow was aperiodic in this range. Later, Dryden and Heald (1926) also registered a rapid increase in the frequency with the Reynolds number when the flow is in the critical zone. Delany and Sorensen (1953) experimented on cylinders of different shapes. Although the data of vortex shedding frequencies in the circular cylinder were not exhaustive and much more scattered than for drag coefficient, they reported non- dimensional frequencies around 0.35 and 0.45 in the range of $R e=10^{6}-2 \times 10^{6}$. Roshko (1961), in his experiments in the high super-critical and trans-critical regimes, for $R e<3.5 \times 10^{6}$ did not observe any periodic behaviour whatsoever. Comparing his results with those of Delany and Sorensen (1953), he attributed the inconsistency in his measurements to the position of the probe used, which was located at $[x, y] \equiv[7.3 D, 0.7 D]$ off the wake centreline.

Jones (1968) carried out measurements in the range $R e=3.6 \times 10^{5}-18 \times 10^{6}$ and distinguished different regimes: a wide-band random regime in the high super-critical regime at $R e=1.1 \times 10^{6}-3.5 \times 10^{6}$ where no dominant frequency was detected, a narrow-band random regime in the trans-critical zone at $R e=3.5 \times 10^{6}-6 \times 10^{6}$ and a quasi-periodic regime at larger Reynolds numbers with characteristic non-dimensional frequency of $S t=$ 0.3. Bearman (1969) studied the vortex shedding in the critical and super-critical regimes. He found that as the fluid enters the critical zone the Strouhal number increases, being 0.32 in the asymmetric zone to rise up to 0.46 in the symmetric super-critical zone. These 
measurements were later confirmed by Achenbach and Heinecke (1981) who also detected vortex shedding in the super-critical zone with a value a bit larger of $S t=0.5$, however. They attributed the differences to the blockage ratio of the wind tunnel which was smaller in the experiments of Bearman (1969). The measurements of Schewe (1983) demonstrated that the asymmetries in the flow in the critical regime reported in previous works (e.g Bearman, 1969) were of a fundamental nature. He did also register vortex shedding in the whole super-critical zone with a non-dimensional vortex shedding frequency of $S t=0.48$, close to those measured by Bearman (1969) and Achenbach and Heinecke (1981). Other experimental results reported in the literature attempted to measure vortex shedding at these Reynolds numbers (Van Nunen, 1974; Shih et al., 1993) but did not succeed in capturing the fundamental frequency in the super-critical regime. It is not clear why there is that large scattering in the detection and measuring of the wake vortex shedding, but different authors have reported that substantial changes might appear by the influence of axial or geometrical perturbations, thus altering the way the wake develops (Fung, 1960; Schmidt, 1965; Achenbach and Heinecke, 1981).

With the rapid development of numerical techniques in the last two decades, the use of computational fluid dynamics at high Reynolds numbers is becoming feasible and a complement to experimental studies. In that sense, large eddy simulations (LES) of the flow are an interesting tool to cope with the complex fluid behaviour at critical Reynolds numbers and beyond. Breuer (2000) analysed the capabilities of LES for solving the flow past a cylinder at the near-critical Reynolds number of $R e=1.4 \times 10^{5}$ with rather good results. Karabelas (2010) did also solve the same Reynolds number but for both the stationary and rotating cylinders, with good agreement with the experimental results for the non-rotating one. Other researchers (Catalano et al., 2003; Moussaed et al., 2014) have also shown the possibilities of LES to capture the drop in the drag in the super-critical regime. In a previous work (Lehmkuhl et al., 2014), by means of LES computations, the flow in the critical regime was studied and fundamental traits of the flow in this regime; e.g. measurements of the size of the laminar separation bubble, drag and lift coefficients, amongst others; were reported.

In the present work, a further insight into the characteristics of the flow in both the 
critical and super-critical regimes is presented by analysing the wake topology and the variation of different critical parameters such as the vortex formation length, recirculation length, wake width, amongst others. Furthermore, vortex shedding and fluctuating forces are studied, giving a more complete picture of the variations the flow experiences when goes from the critical to the super-critical regime. Results obtained are compared (when available) to experimental results.

\section{Mathematical and numerical model}

The spatially filtered incompressible Navier-Stokes equations can be written as,

$$
\begin{array}{r}
\frac{\partial \bar{u}_{i}}{\partial x_{i}}=0 \\
\frac{\partial \bar{u}_{i}}{\partial t}+\frac{\partial \bar{u}_{i} \bar{u}_{j}}{\partial x_{j}}-\nu \frac{\partial^{2} \bar{u}_{i}}{\partial x_{j} \partial x_{j}}+\rho^{-1} \frac{\partial \bar{p}}{\partial x_{i}}=-\frac{\partial \mathcal{T}_{i j}}{\partial x_{j}}
\end{array}
$$

where $x_{i}$ and $t$ are the spatial position in the $i$ direction and the time, respectively. $\bar{u}_{i}$ and $\bar{p}$ stand for the filtered velocity and pressure. $\nu$ is the kinematic viscosity and $\rho$ the density of the fluid. In equation 2, $\mathcal{T}_{i j}$ is the subgrid scale (SGS) stress tensor which has to be modelled. Its deviatoric part is given by,

$$
\mathcal{T}_{i j}-\frac{1}{3} \mathcal{T}_{k k} \delta_{i j}=-2 \nu_{s g s} \overline{\mathcal{S}}_{i j}
$$

where $\overline{\mathcal{S}}_{i j}$ is the large-scale rate-of-strain tensor, $\overline{\mathcal{S}}_{i j}=\frac{1}{2}\left(g_{i j}+g_{j i}\right)$ being $g_{i j}=\partial \bar{u}_{i} / \partial x_{j}$. $\delta_{i j}$ is the Kronecker delta. The formulation is closed by formulating an appropriate expression for the subgrid-scale viscosity, $\nu_{s g s}$. In this work, the wall-adapting local-eddy viscosity model (WALE) (Nicoud and Ducros, 1999) is used. The WALE model evaluates the eddy viscosity as,

$$
\nu_{s g s}=\left(\mathcal{C}_{w} \Delta\right)^{2} \frac{\left(\overline{\mathcal{V}_{i j}}: \overline{\mathcal{V}_{i j}}\right)^{\frac{3}{2}}}{\left.\left(\overline{\mathcal{S}_{i j}}: \overline{\mathcal{S}_{i j}}\right)^{\frac{5}{2}}+\overline{\left(\mathcal{V}_{i j}\right.}: \overline{\mathcal{V}_{i j}}\right)^{\frac{5}{4}}}
$$


where, $\overline{\mathcal{V}_{i j}}$ is the deviatoric part of the square of the velocity gradient tensor $\overline{\mathcal{V}_{i j}}=$ $\frac{1}{2}\left(g_{i j}^{2}+g_{j i}^{2}\right)-\frac{1}{3} \delta_{i j} g_{k k}^{2}$, with $g_{i j}^{2}=g_{i k} g_{k j}$, and $\mathcal{C}_{w}$ is the model constant. Here a value of $\mathcal{C}_{w}=0.325$ is used.

The governing equations are discretised on a collocated unstructured grid arrangement by means of second-order spectrum-consistent schemes (Verstappen and Veldman, 2003). Such schemes are conservative, i.e. they preserve the symmetry properties of the continuous differential operators, and ensure both stability and conservation of the kinetic-energy balance even at high Reynolds numbers and with coarse grids. For the temporal discretisation of the momentum equation a two-step linear explicit scheme on a fractional-step method is used for the convective and diffusive terms (Trias and Lehmkuhl, 2011), while for the pressure gradient term an implicit first-order scheme is implemented. The Poisson system derived from the incompressibility constraint is solved by means of a FFT-based direct solver (Borrell et al., 2011). For more details about the numerical method, the user is referred to Rodríguez et al. (2011); Jofre et al. (2014); Trias et al. (2014).

\subsection{Definition of the case and boundary conditions}

The flow past a circular cylinder at critical and super-critical Reynolds numbers in the range of $R e=U_{\text {ref }} D / \nu=2.5 \times 10^{5}-8.5 \times 10^{5}$ is considered. The Reynolds number is defined in terms of the free-stream velocity $U_{\text {ref }}$ and the cylinder diameter $D$. For all the cases, the circular cylinder is placed at $(x, y, z) \equiv(0,0,0)$, the dimensions of the computational domain being of $x \equiv[-16 D, 16 D] ; y \equiv[-10 D, 10 D]$ for the stream- and cross-stream directions, whereas for the span-wise direction $z \equiv[0,0.5 \pi D]$ is considered for Reynolds number up to $R e=6.5 \times 10^{5}$ and $z \equiv[0, D]$ for the higher Reynolds numbers (see table 1). Being $L_{y}$ the size of the computational domain in the cross-stream direction, the blockage ratio is $D / L_{y}=5 \%$. In Table A.4 in the AppendixA, a compilation of the measurement conditions for most of the experimental data used for comparison in this work is given. As can be seen, the blockage ratio used in the present computations is well within the values reported in the literature. It should be borne in mind that blockage ratio may affect the results as this magnitude increases. West and Apelt (1981) showed that blockages lower than 6\% has 
Table 1: Computational meshes $N C V_{t}$ total number of CVs; $N_{C V}$ plane number of CVs in the plane; $N_{\text {planes }}$ number of planes in the span-wise direction; $L_{z}$ is the size of the span-wise direction.

\begin{tabular}{ccccc}
\hline$R e$ & $N C V_{t}\left[\times 10^{6}\right]$ & $N_{C V \text { plane }}$ & $N_{\text {planes }}$ & $L_{z}$ \\
\hline $2.5 \times 10^{5}$ & 38.4 & 299683 & 128 & $0.5 \pi D$ \\
$3.8 \times 10^{5}$ & 48.6 & 379950 & 128 & $0.5 \pi D$ \\
$5.3 \times 10^{5}$ & 64.1 & 500516 & 128 & $0.5 \pi D$ \\
$6.5 \times 10^{5}$ & 83.2 & 650432 & 128 & $0.5 \pi D$ \\
$7.2 \times 10^{5}$ & 89.4 & 698949 & 128 & $D$ \\
$8.5 \times 10^{5}$ & 105.1 & 820803 & 128 & $D$ \\
\hline
\end{tabular}

negligible effects on pressure distribution and drag. For larger blockages, the general effect, at least in the sub-critical range, is to increase the force coefficients as well as the span-wise correlation (Blackburn, 1994). In general, blockage may affect separation due to changes on the surface pressure gradients, thus triggering the critical transition at lower Reynolds numbers. Nonetheless, as pointed out by Blackburn and Melbourne (1996), these effects would be of less importance in the super-critical regime as the wake width is smaller.

In the present computations the boundary conditions consist of uniform velocity $(u, v, w)=$ $(1,0,0)$ at the inflow, symmetry conditions at the top and bottom boundaries of the domain, while at the outlet a pressure-based condition is used. At the cylinder surface, no-slip conditions are prescribed. As for the span-wise direction, periodic boundary conditions are imposed.

\subsection{Numerical domain. Mesh resolution studies.}

Different unstructured grids from 38 million up to $\sim 105$ million control volumes are used for solving the different cases depending on the Reynolds number (see table 1). It was shown in Lehmkuhl et al. (2014) that in order to capture the complexities of the physical 


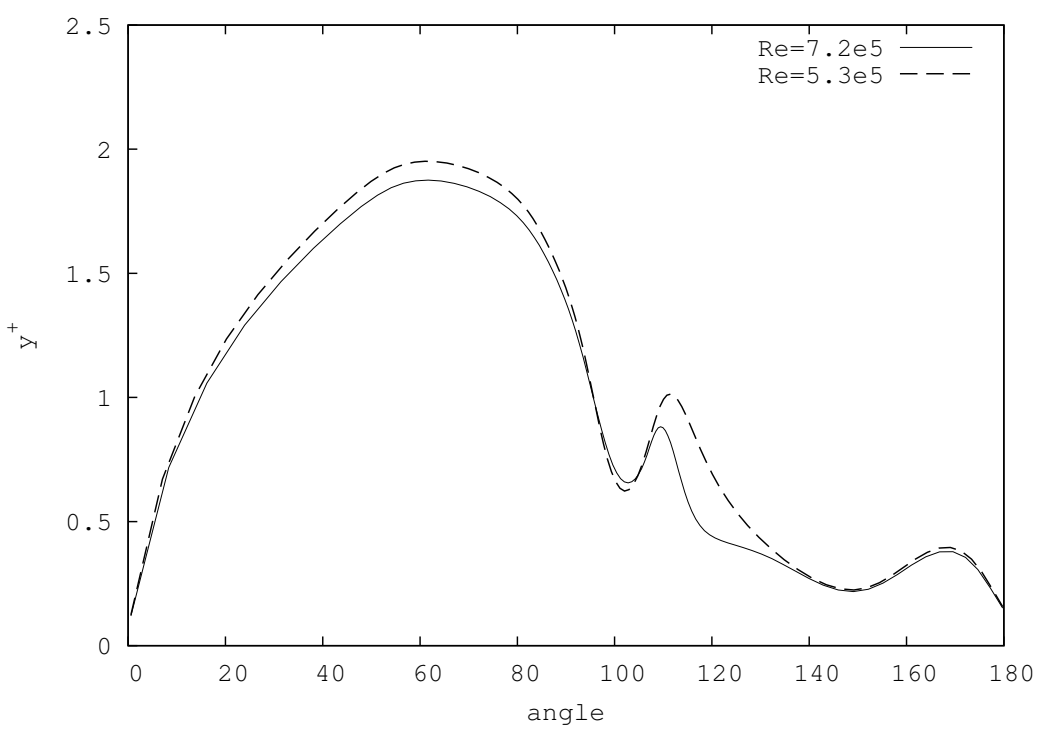

Figure 1: Mesh resolution near the wall for $R e=5.3 \times 10^{5}$ and $R e=7.2 \times 10^{5}$.

phenomenon in the zone close to the cylinder; i.e. delayed separation, transition to turbulence upon separation, reattachment, and further turbulent separation, amongst others; the boundary layer should be well-resolved. To that end, control volumes are clustered towards the cylinder wall and, a prism layer is constructed around the cylinder so as the non-dimensional wall distance is kept $y^{+}<2$. Being $u_{\tau}=\sqrt{\tau_{w} / \rho}$ the friction velocity and $n$ the wall-normal distance, the non-dimensional wall-normal distance along the cylinder circumference is evaluated as $y^{+}=u_{\tau} n / \nu$. The non-dimensional wall-normal distance for $R e=5.3 \times 10^{5}$ and for $R e=7.2 \times 10^{5}$ is plotted in figure 1.

Regarding the span-wise size of the domain, for Reynolds numbers $R e \leq 6.5 \times 10^{5}$ a length of $L_{z}=0.5 \pi D$ was enough to capture the largest scales of the flow (see Lehmkuhl et al. (2014) for more details). Notice that this value is lower than those used in experimental set-ups (see AppendixA). However in experiments, contrary to numerical computations, the combination of cylinder end conditions and aspect ratio $L_{z} / D$ are of importance since structures formed close to the wall may interact with the vortex shedding and trigger the wake three-dimensionality (this issue will be addressed in more detail in section 3.2). This is especially true for the flow at critical and super-critical regimes. Thus a relative large 


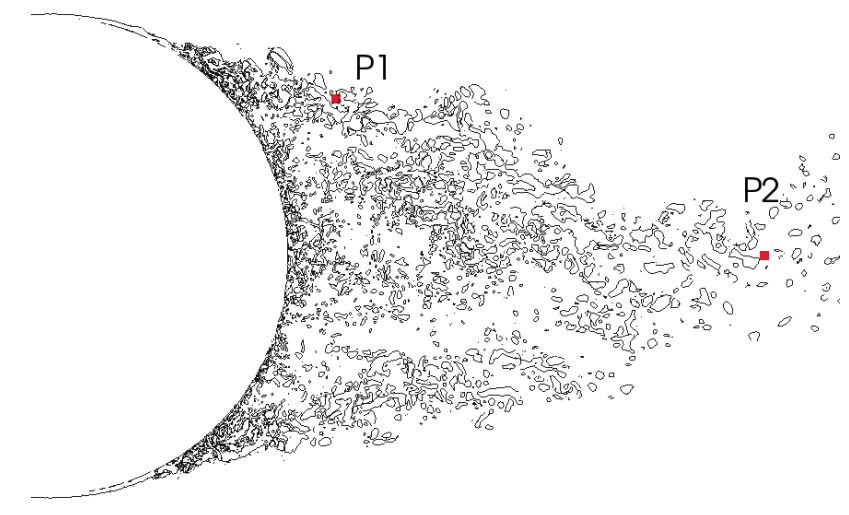

Figure 2: Location of the numerical probes: $P 1 \equiv(0.6 D, 0.33 D)$ and $P 2 \equiv(1.3 D, 0)$.

aspect ratio is necessary so as to keep the measurement zone at the cylinder mid-span unaffected by the end plates and tunnel walls. In fact, in some experimental studies this issue has been addressed; for instance in the work of Bearman (1969) the variation of the base pressure along the cylinder span is measured in order to study the influence of the end-wall boundary layers on the measurements. However, as in the present computations periodic boundary conditions in the span-wise direction are set, one just must focus on guaranteeing that the actual span-wise size is enough so as to contain all the relevant structures of the flow. Thus based on the results of two-point correlations at different positions in the near wake, the domain was considered large enough (Lehmkuhl et al., 2014). This was also in agreement with previous experimental observations. Humphreys (1960), by means of indirect measurements using silk threads, estimated a correlation length between 1.4D and 1.7D for Reynolds numbers between $10^{5}$ and $3.5 \times 10^{5}$. In the range of $R e=3.8 \times 10^{5}-7.5 \times 10^{5}$, Schmidt (1965) found correlation lengths for drag and lift forces of less than 1D. This is also in agreement with correlation coefficients measured by Spitzer (1965) which were found to be lower than $1 \mathrm{D}$ for $R e>5.3 \times 10^{5}$ and about $1 \mathrm{D}$ for $R e<5.3 \times 10^{5}$.

In the light of these results, for the super-critical Reynolds numbers of $R e=7.2 \times 10^{5}$ and $R e=8.5 \times 10^{5}$ the span-wise size is set to $L_{z}=D$ (see table11). Nonetheless, two-point 


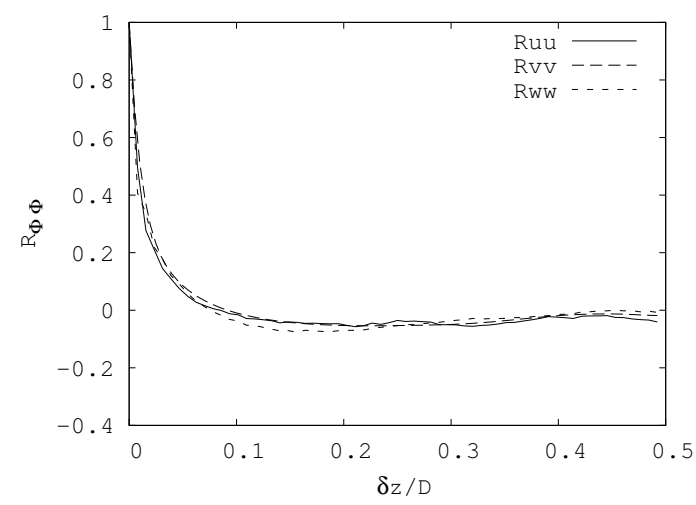

(a)

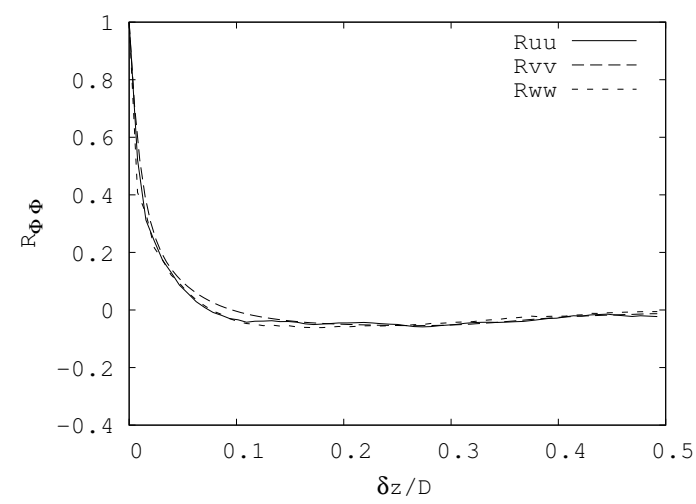

(c)

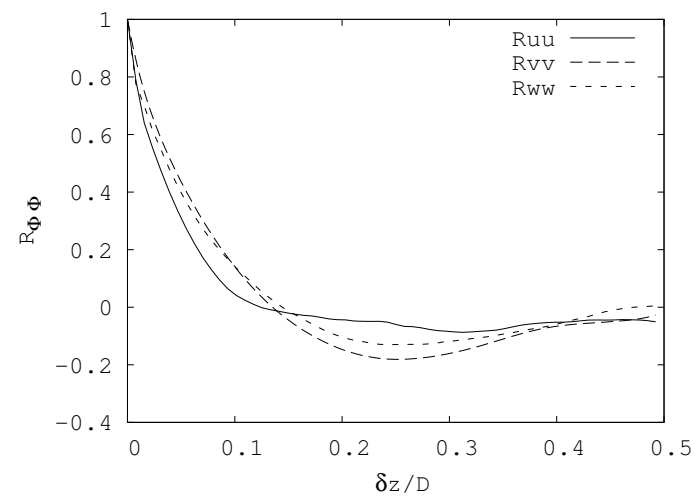

(b)

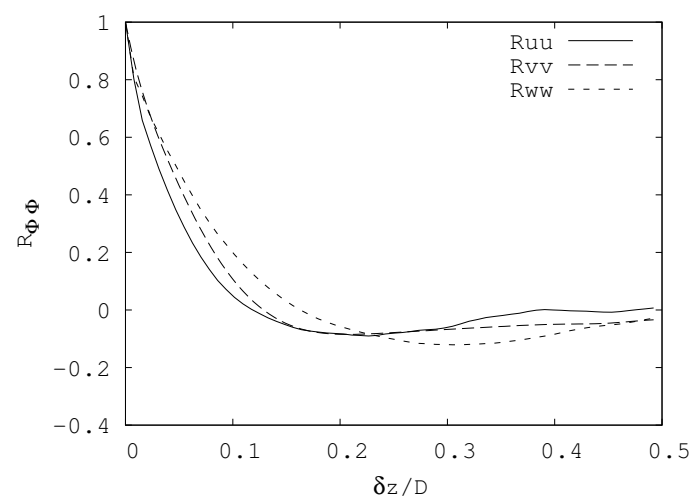

(d)

Figure 3: Two-point correlations of the stream-wise, cross-stream and span-wise velocity fluctuations in the near wake $(\mathrm{a}, \mathrm{b})$ at $R e=7.2 \times 10^{5}$ at $(x / D, y / D) \equiv(0.6,0.33)$ and $(x / D, y / D) \equiv(1.3,0)$, respectively; $(\mathrm{c}, \mathrm{d})$ at $R e=8.5 \times 10^{5}$ at $(x / D, y / D) \equiv(0.6,0.33)$ and $(x / D, y / D) \equiv(1.3,0)$, respectively 
correlations at different locations of the near wake are here evaluated in order to check if this size is large enough to accommodate all structures. The locations where these correlations are evaluated are shown in figure 2. These locations are: $P 1 \equiv(0.6 D, 0.33 D)$ in the turbulent separated shear layer and $P 2 \equiv(1.3 D, 0)$ in the wake centreline. One-dimensional two-point correlations of the fluctuating variable $\phi^{\prime}=\phi-\langle\phi\rangle$,

$$
\Re_{\phi \phi}(x, y, \delta z)=\frac{\left\langle\phi^{\prime}(x, y, z, t) \phi^{\prime}(x, y, z+\delta z, t)\right\rangle}{\left\langle\phi^{\prime 2}\right\rangle}
$$

are plotted in figure 3. In the above equation, $\langle\cdot\rangle$ denotes averaging over time. In the detached turbulent boundary layer (see figure [3a,c), correlation coefficients drop to zero much faster than in the wake centreline, where larger structures are observed (figure 3b,d). Nonetheless, correlation values for all three velocity fluctuations fall-off to zero for separations lower than half of the domain. Thus, a span-wise length of $1 \mathrm{D}$ seems to be acceptable for these Reynolds numbers.

\section{Results}

\subsection{Wake topology}

Once the flow enters the critical regime, a laminar separation bubble (LSB) is formed on one side of the cylinder surface as a consequence of the combined effects of flow separation, turbulence transition in the separated shear-layer and further reattachment of the flow (see Lehmkuhl et al. (2014) at $\left.R e=2.5 \times 10^{5}\right)$. However, as transition to turbulence is asymmetric, the flow on the other side of the cylinder remains unaffected as in the sub-critical regime. As a result, the forces acting on the cylinder surface are also asymmetric and the magnitude of the steady lift coefficient is greater than zero. With the increase in the Reynolds number a second bubble starts to form, yet the flow is asymmetric $\left(R e=3.8 \times 10^{5}\right)$. Flow symmetry is reached when the second bubble stabilises at the end of the critical regime $\left(R e \approx 5.3 \times 10^{5}-6.5 \times 10^{5}\right)$ and the drop in the drag is completed. The complete formation of this second bubble and the symmetry recovery marks the onset of the super-critical regime. 


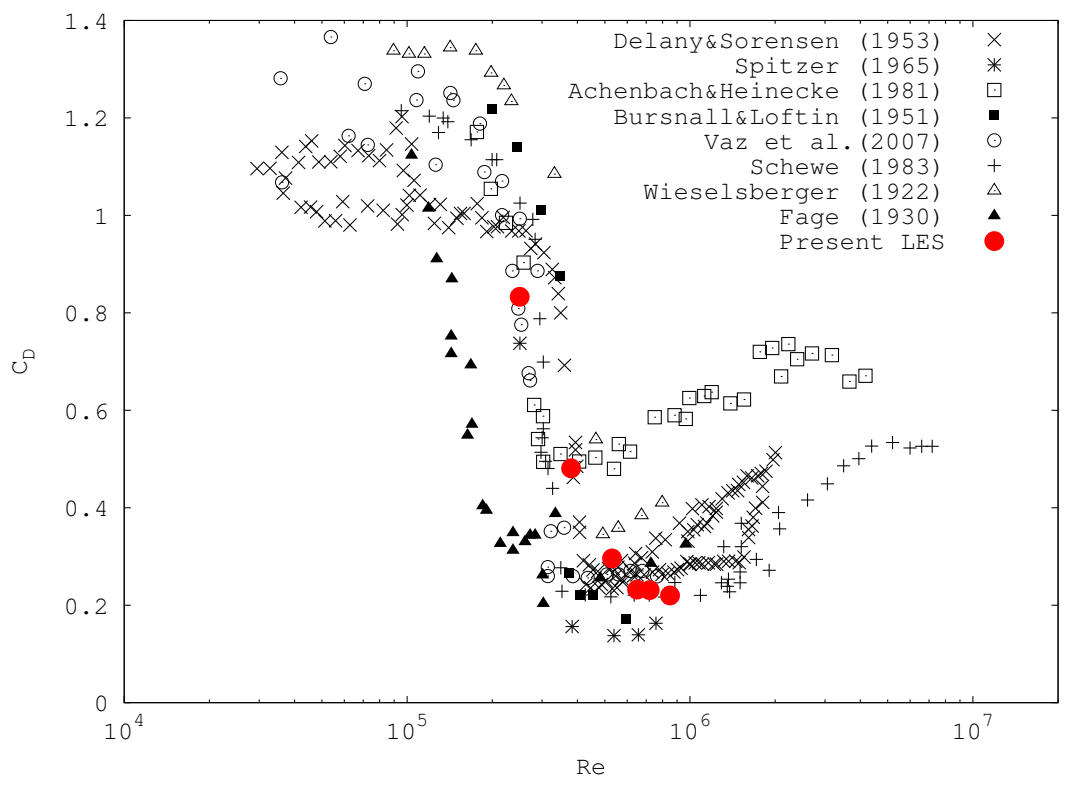

Figure 4: Critical and super-critical drag coefficient. Comparison with the literature. (๑) Present results; (×) Delanv and Sorensen (1953); (*) Spitzer (1965); (๑)

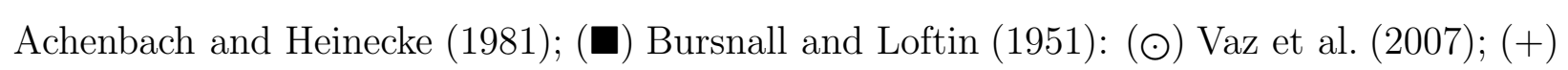
Schewe (1983); ( $\triangle$ ) Wieselsberger (1922); ( $)$ Fage (1930). 
In figure 4, the drag coefficient at both critical and super-critical Reynolds numbers is plotted together with available experimental measurements from the literature. The agreement is rather good; the present computations are capable of capturing the steep drop in the drag at critical Reynolds numbers and the plateau behaviour observed at super-critical Reynolds numbers, once the drag has dropped to its minimum value. Yet, we would like to draw attention to the somewhat large scattering present in the experimental data, especially at critical Reynolds numbers. These differences may be due to several causes such as compressibility, surface roughness, cylinder end conditions, inlet turbulence intensity, blockage ratio, amongst others.

The influence of the blockage ratio has been addressed in section 2.1. As it has been commented, ratios larger than 6\% (West and Apelt, 1981) might affect the force coefficients. Furthermore, the critical transition might be forced to occur at lower Reynolds numbers. On the other hand, turbulence intensity also induces critical transition at lower Reynolds numbers (Blackburn and Melbourne, 1996). However, the published experimental data (see details summarised in AppendixA used in this work were mainly obtained on low turbulence intensity conditions, probably with the exception of the experiments of Fage (1930). Despite the fact that in Fage's experiments turbulence level was not reported, one may assume they were performed at a higher stream turbulence level since the critical Reynolds number is too low (see figure 4).

An issue of major importance is the compressibility effects that might be encountered even at low Mach numbers, well below the critical value depending on the boundary layer condition, and which occurs near $M a=0.4$ (Achenbach and Heinecke, 1981; Polhamus, 1984). Compressibility, due to the increase in the value of the minimum pressure point which affects separation, shifts the critical transition range towards higher Reynolds numbers. This results in an increased drag at all Reynolds numbers. According to Polhamus (1984), these effects begin to be measurable at $M a=0.18-0.2$, which is also in agreement with measurements of Zan (2008) in the super-critical region. Even though most of the experiments summarised in table A.4 are in the low-Mach region, in some of them $M a>0.2$ conditions are attained, especially for the larger Reynolds numbers e.g. Delany and Sorensen 
(1953); Jones (1968); Shih et al. (1993).

Regarding the cylinder surface conditions, all the experimental results used for comparison in this work were on smooth highly polished cylinders, as can be seen in the AppendixA. According to Szechenyi (1975), below the super-critical regime, smooth cylinders behaves as they have a very small non-dimensional surface roughness equivalent to $k_{s} / D \sim 3.5 \times 10^{-5}$ ( $k_{s}$ is the total height of the surface roughness). In any case, roughness promotes an earlier transition to a turbulent boundary layer, thus the critical regime occurs at smaller Reynolds numbers, whereas minimum drag coefficient increases. However, as the Reynolds number increases and the boundary layer becomes thinner, the value at which a surface may be considered smooth decreases. For instance, Polhamus (1984) pointed out that values of $k_{s} / D \sim 10^{-5}$, as those of Roshko (1961)), can produce a measurable increase in the drag in the super-critical regime.

Apart from the aforementioned effects, discrepancies may also arise due to end flow leakages (Delany and Sorensen, 1953) and latex end seals (Vaz et al., 2007), the use of a three-dimensional cylinder model (Schmidt, 1965); Spitzer, 1965), problems with measurement instruments such as pressure transducers working at a small fraction of their full scale (Shih et al., 1993) and tunnel vibrations at some Reynolds numbers (Schewe, 1983), amongst others.

The local pressure distribution along the cylinder circumference at different Reynolds numbers is given in figure 5. In the figure, angular positions are measured in clock-wise direction. At $R e=2.5 \times 10^{5}$ the pressure distribution is asymmetric (figure $5 \mathrm{a}$ ). Notice that on the top side of the cylinder $\left(\right.$ angle $\left.=0-180^{\circ}\right)$, the agreement with the pressure distribution measured by Cantwell and Coles (1983) at $R e=1.4 \times 10^{5}$ is rather good, especially the magnitude and position of the pressure minimum. This is because on this side of the cylinder the flow resembles that of the sub-critical regime. On the other side of the cylinder $\left(\right.$ angle $=180-360^{\circ}$ ), the flow behaviour is different and a large depression can be observed, with a displacement in the position of the pressure minimum towards the cylinder rear. With the increase in the Reynolds number, changes in the pressure distribution occur on both sides of the cylinder and symmetry is gradually recovered (e.g. $R e=5.3 \times 10^{5}$ in 


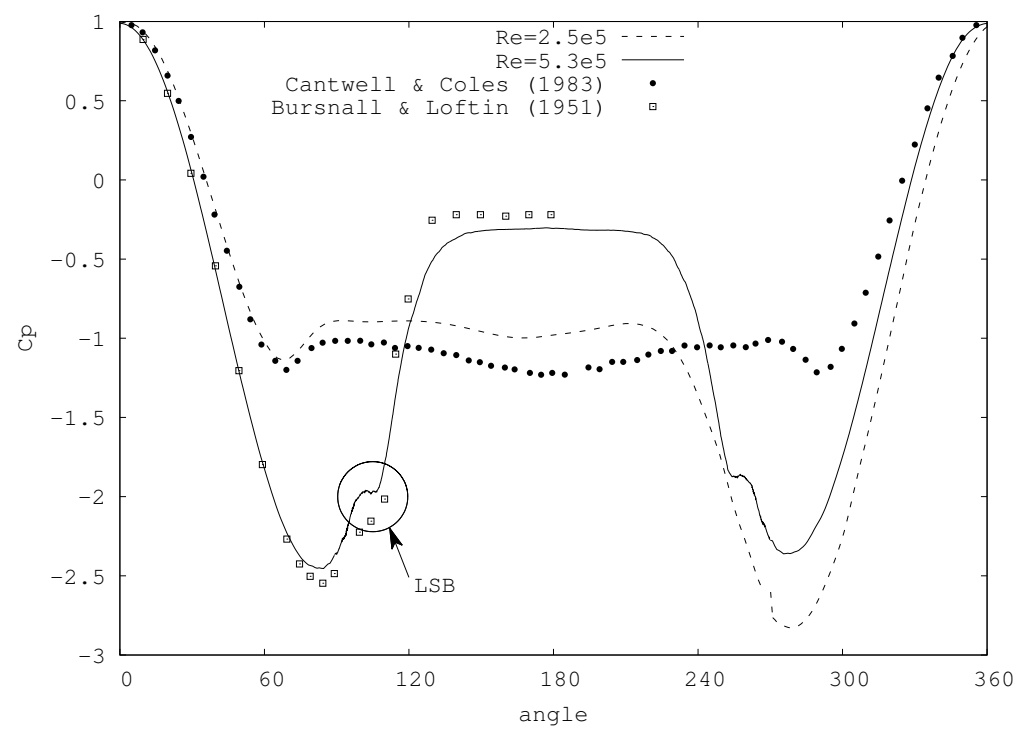

(a)

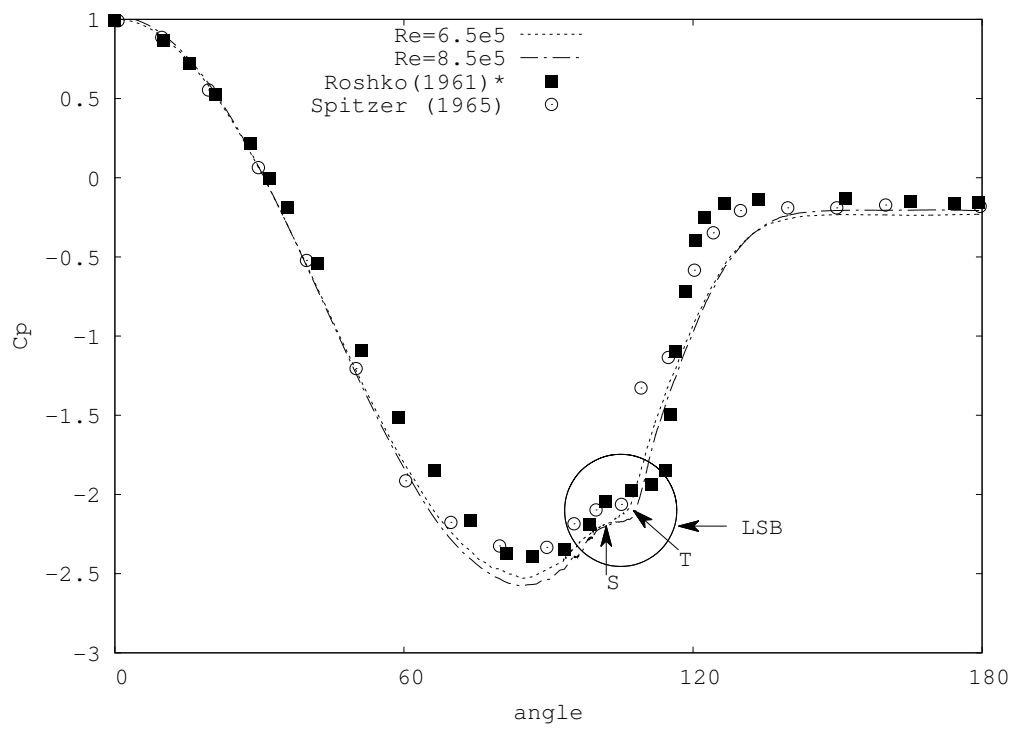

(b)

Figure 5: Local pressure distribution at different Reynolds numbers. Comparison with the literature. (a) At critical Reynolds numbers $R e=2.5 \times 10^{5}$ and $R e=5.3 \times 10^{5}$; (b) At super-critical Reynolds numbers of $R e=6.5 \times 10^{5}$ and $R e=8.5 \times 10^{5}$. * Results by Spitzer (1965) at $R e=7.5 \times 10^{5}$. ${ }^{*}$ Flachsbart at $R e=6.7 \times 10^{5}$ taken from Roshko (1961). Notice that for $R e=6.5 \times 10^{5}$ the approximate location of the laminar separation of the LSB $(\mathrm{S})$ and transition to turbulence $(\mathrm{T})$ are also indicated. 
Table 2: Flow parameters. Drag coefficient $C_{D}$; fluctuating $\operatorname{drag} C_{D, r m s}$; lift coefficient $C_{L}$; fluctuating lift $C_{L, r m s}$; cylinder base pressure $-C_{p, b a s e}$.

\begin{tabular}{cccccc}
\hline$R e$ & $C_{D}$ & $C_{D, r m s}$ & $C_{L}$ & $C_{L, r m s}$ & $-C_{p, \text { base }}$ \\
\hline $2.5 \times 10^{5}$ & 0.833 & 0.095 & -0.903 & 0.486 & 0.99 \\
$3.8 \times 10^{5}$ & 0.481 & 0.061 & 0.245 & 0.217 & 0.48 \\
$5.3 \times 10^{5}$ & 0.296 & 0.011 & 0.0614 & 0.0705 & 0.305 \\
$6.5 \times 10^{5}$ & 0.232 & 0.0081 & 0.027 & 0.0755 & 0.23 \\
$7.2 \times 10^{5}$ & 0.213 & 0.0094 & 0.016 & 0.0752 & 0.224 \\
$8.5 \times 10^{5}$ & 0.218 & 0.0092 & 0.007 & 0.070 & 0.207 \\
\hline
\end{tabular}

figure $5 \mathrm{a}$ ). These changes can be summarised as a decrease in the magnitude of the minimum pressure, an increase of base pressure and the formation of a laminar separation bubble on the cylinder rear, identified as a small plateau in the pressure distribution at angles about $\phi \sim 100^{\circ}-110^{\circ}$. In figure [5b, as the pressure distribution is symmetric at super-critical Reynolds numbers, only one side of the cylinder is represented. Notice that the approximate location of the boundary layer laminar separation $(\mathrm{S})$ and transition to turbulence $(\mathrm{T})$ for $R e=6.5 \times 10^{5}$ are marked in the figure. For the other Reynolds numbers depicted similar positions can be identified, but for the sake of clarity they were not marked in the figure. The angular size of the LSB decreases slightly as the Reynolds number increases being at the super-critical $R e=8.5 \times 10^{5}$ about $8^{\circ}$, whereas at the end of the critical regime at $R e=5.3 \times 10^{5}$ it spans about $15^{\circ}$.

The average magnitude and fluctuations of drag and lift coefficients and the base pressure are given in table 2. The high value of the lift coefficient observed at $R e=2.5 \times 10^{5}$ is a signature of the asymmetric forces acting on the cylinder. The fluctuating lift, which is about an order of magnitude larger than the fluctuating drag, also presents a large value at this Reynolds number, but decreases up to a value about $C_{L, r m s} \sim 0.07$ to remain almost constant along the super-critical regime. We will return to this point in detail in the next 


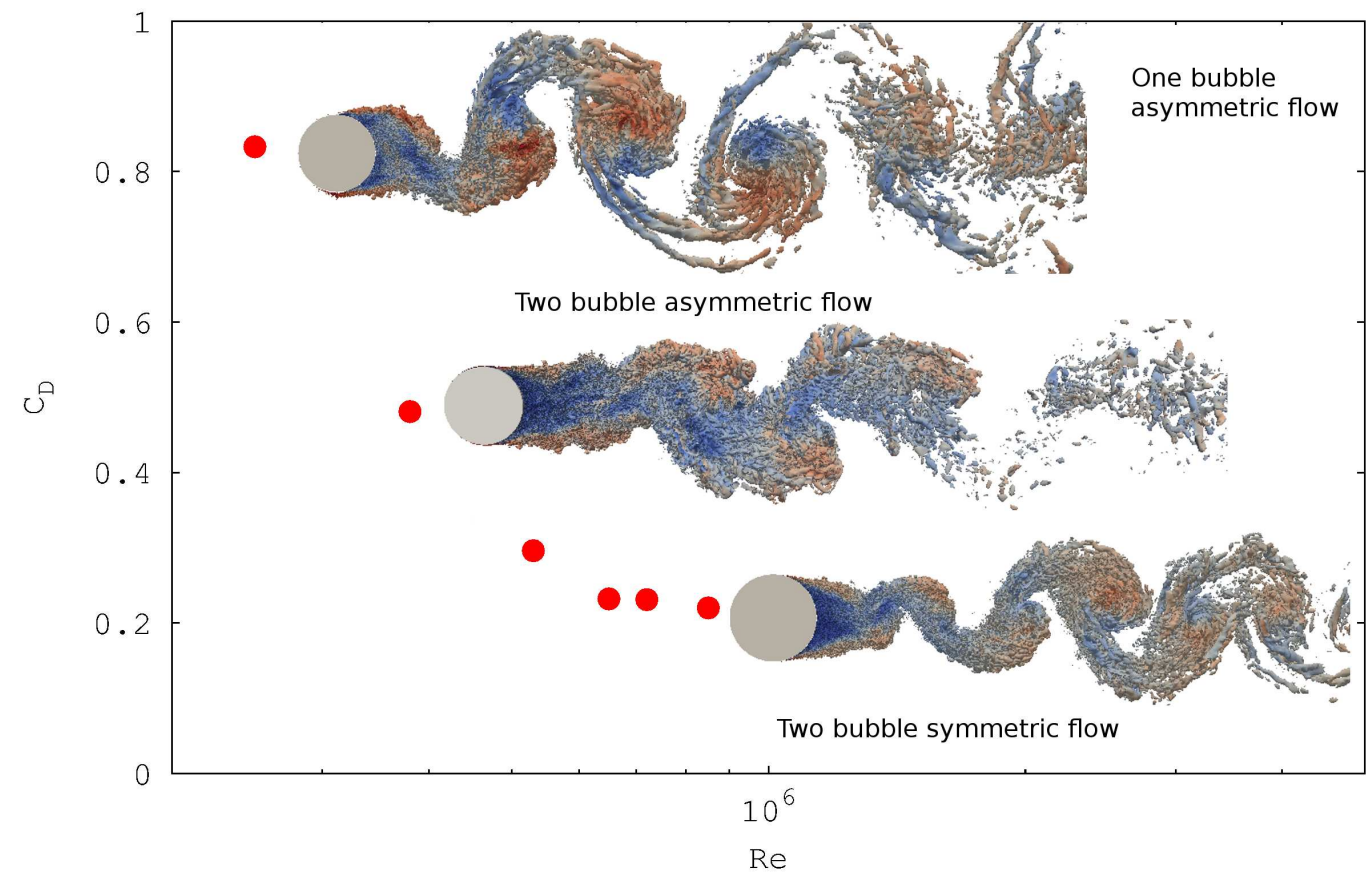

Figure 6: Different wake configurations observed in the critical and super-critical regimes.

section.

According to the changes in the symmetry of the flow observed (see also Lehmkuhl et al., 2014), three modes can be foreseen (figure 6): i) a one-bubble asymmetric mode, with flow separation delayed on one side of the cylinder while the other remains sub-critical; ii) a two-bubble asymmetric mode, with a small second bubble starting to be formed and; iii) a two-bubble symmetric mode, with two symmetric bubbles on each side of the cylinder which is a characteristic trait of the super-critical regime. As it will be shown hereafter, the flow in the third mode is stable and little changes are observed in the wake characteristics.

In figure 7, the mean streamlines in the near wake region are depicted for all Reynolds numbers. A two-eddy configuration pattern is observed at all Reynolds numbers, though differences in size, shape and length can readily be identified. The position of (laminar or turbulent) flow separation $\phi_{s}$ and the vortex centroids are marked for each case in the figure. At $R e=2.5 \times 10^{5}$ (figure $7 \mathrm{~b}$ ), recirculation vortices are of different size and streamlines are bent towards the opposite side where the LSB is formed, i.e. the side with the lower pressure 


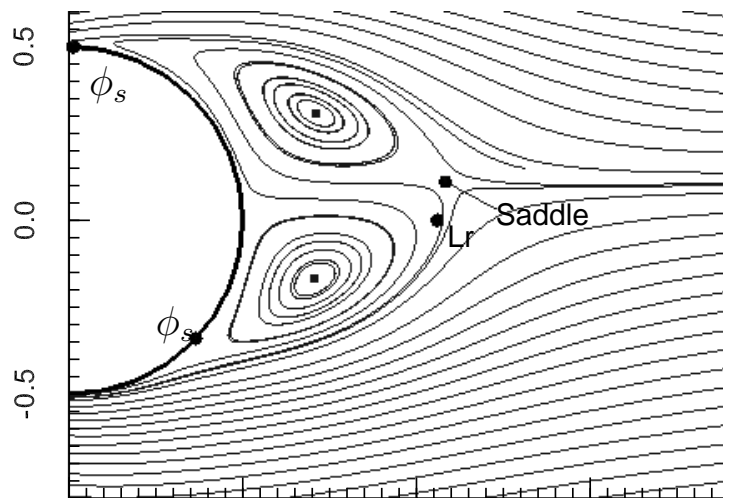

0.0

0.5

(a)

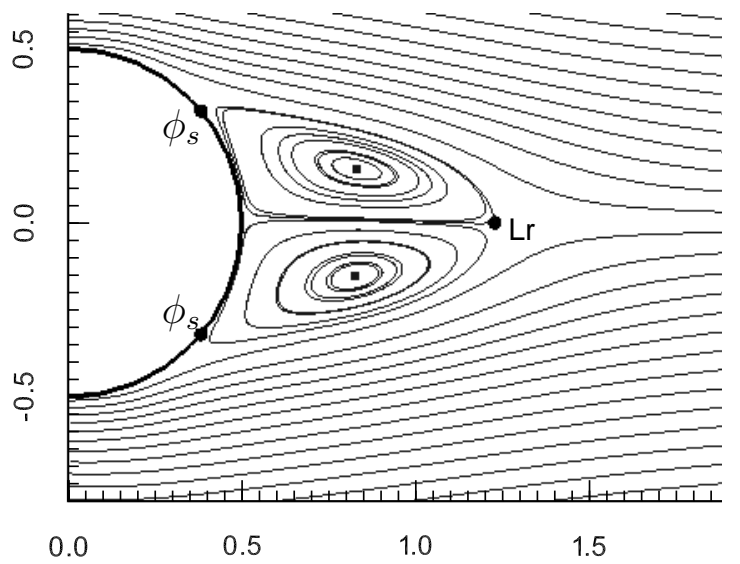

(c)

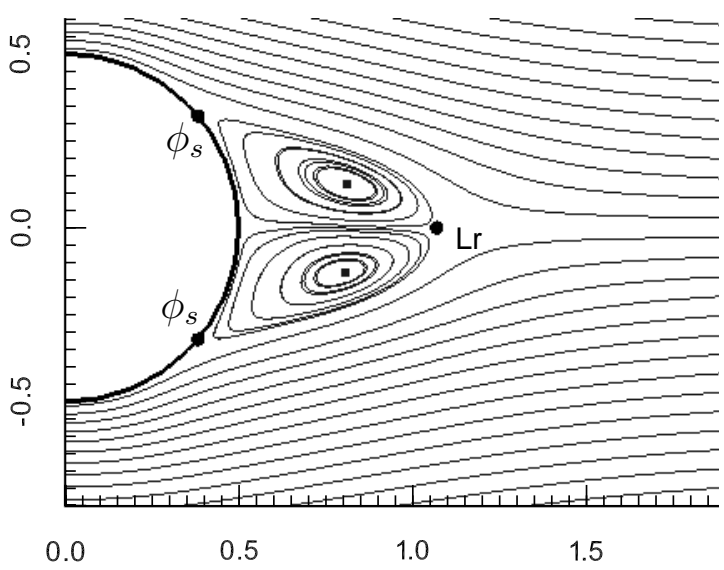

(e)

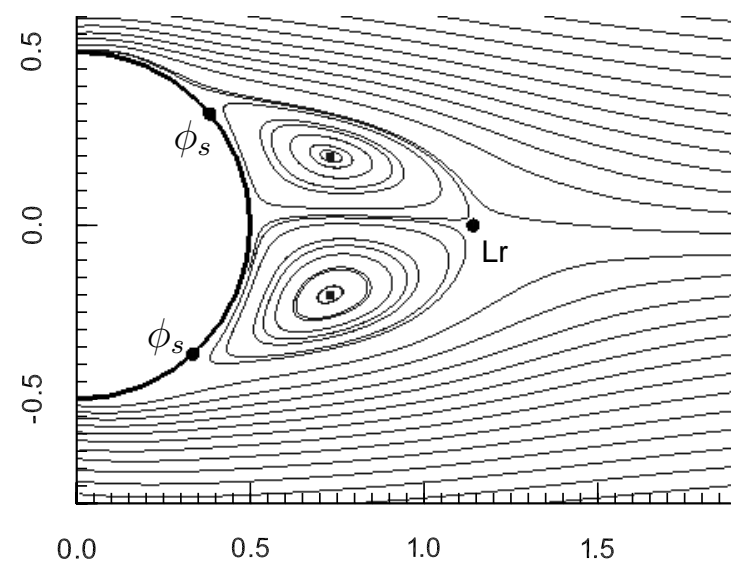

(b)

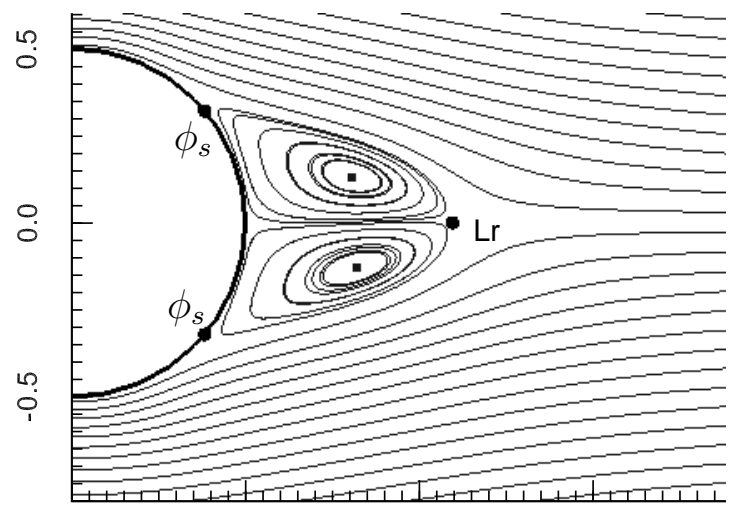

$\begin{array}{llll}0.0 & 0.5 & 1.0 & 1.5\end{array}$

(d)

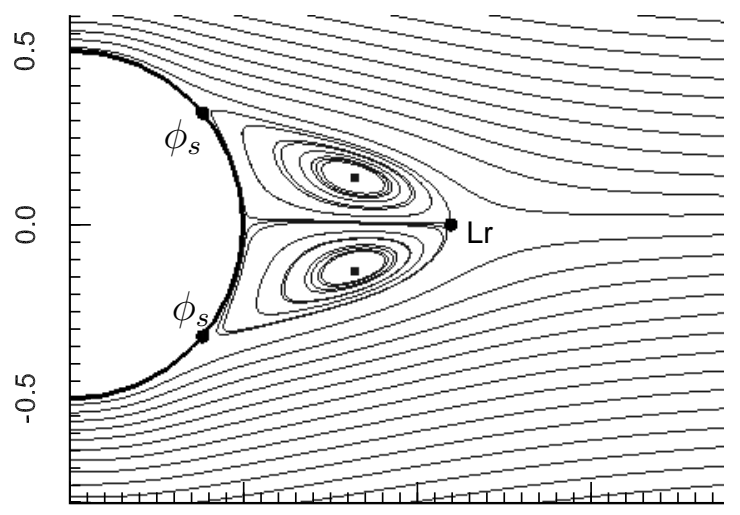

$\begin{array}{llll}0.0 & 0.5 & 1.0 & 1.5\end{array}$

(f)

Figure 7: Flow average streamlines. (a) at $R e=2.5 \times 10^{5}$; (b) at $R e=3.8 \times 10^{5}$; (c) at $R e=5.3 \times 10^{5}$; (d) at $R e=6.5 \times 10^{5}$; (e) at $R e=7.2 \times 10^{5}$ ); (f) at $R e=8.5 \times 10^{5}$. 
gradient. This is a consequence of the difference in magnitude of the forces acting on both sides of the cylinder. In this case, streamlines are bent towards the upper part of the wake (see also figure 5a). The top eddy is similar to those seen in the sub-critical flow with laminar separation close to the cylinder apex $\phi_{s} \sim 90^{\circ}$. On the other hand, the bottom eddy is smaller, its centroid (defined as in Cantwell and Coles (1983)) is located closer to the wake centreline than the opposite one (the position of the centroid of the eddies is given in table 3i) and turbulent flow separation is shifted towards the cylinder rear $\left(\phi_{s} \sim 218^{\circ}\right)$.

The non-dimensional length of the recirculation zone $L_{r}$ can also be observed from the figure. This is the distance to the position where the recirculation closes, i.e. the distance from the cylinder rear end to the saddle point formed by the time averaged streamline topology. For a symmetric wake, this location is in the plane $[x, y=0]$. However, as for $R e=2.5 \times 10^{5}$ the wake is asymmetric, the saddle point does not sit in this plane but slightly off at $[x, y] \equiv[1.082,0.111]$. In table 3 , the non-dimensional length of the recirculation together with other characteristic length scales such as the non-dimensional vortex formation length, $L_{f}$ (defined as in Bloor and Gerrard (1966), i.e. the distance between the cylinder centroid and the location where the root-mean square stream-wise velocity peaks), and the non-dimensional distance from the cylinder centre to the position where the stream-wise velocity at the wake centreline reaches a minimum value, $L_{u, \min }$, are also given. Notice that in the table all these parameters are given for the cylinder mid-plane $[x, y=0]$, thus the length of the recirculation for $R e=2.5 \times 10^{5}$ was determined from the position where the mean stream-wise velocity is zero. This distance is $L_{r}=0.559$. However, if the distance from the cylinder rear to the location of the saddle is considered, the actual size of the recirculation zone is 0.588 . This value is close to those reported at the end of the subcritical regime at $R e=1.4 \times 10^{5}$, although some scatter in the measurement of this wake length scale can be found in the literature (e.g. $L_{r}=0.6$ in Cantwell and Coles (1983), $L_{r}=0.73$ in Braza et al. (2006), $L_{r} \approx 0.42$ in Breuer (2000)).

In a lower degree, asymmetries in the flow are also present at $R e=3.8 \times 10^{5}$ with the wake bent down, i.e., towards the side with the lower pressure gradient (smaller bubble). This Reynolds number corresponds to an intermediate state where two LSB are formed, but 
Table 3: Critical parameters of the near wake. $L_{r}$, non-dimensional length of the recirculation zone; $L_{f}$, non-dimensional vortex formation length; $L_{u, \min }, u_{\min }$ non-dimensional location and magnitude of the minimum stream-wise velocity $u / U_{\text {ref }}$ in the wake centreline, respectively; $x_{c}$ and $y_{c}$, non-dimensional stream-wise and cross-stream coordinates of the location of the mean wake vortex centre.

\begin{tabular}{ccccccc}
\hline & & & & & \multicolumn{2}{c}{ vortex centre } \\
$R e$ & $L_{r}$ & $L_{f}$ & $L_{u, \min }$ & $u_{\min }$ & $x_{c}$ & $y_{c}$ \\
\hline $2.5 \times 10^{5}$ & 0.559 & 1.105 & 0.757 & -0.194 & $0.711 / 0.707$ & $0.308 /-0.168$ \\
$3.8 \times 10^{5}$ & 0.641 & 1.107 & 0.778 & -0.205 & $0.73 / 0.728$ & $0.199 /-0.2$ \\
$5.3 \times 10^{5}$ & 0.727 & 1.183 & 0.885 & -0.253 & $0.831 / 0.825$ & $0.153 /-0.154$ \\
$6.5 \times 10^{5}$ & 0.596 & 1.044 & 0.86 & -0.262 & $0.807 / 0.822$ & $0.132 /-0.129$ \\
$7.2 \times 10^{5}$ & 0.569 & 1.062 & 0.854 & -0.254 & $0.813 / 0.807$ & $0.125 /-0.128$ \\
$8.5 \times 10^{5}$ & 0.597 & 1.091 & 0.871 & -0.23 & $0.819 / 0.820$ & $0.135 /-0.134$ \\
\hline
\end{tabular}

they are different in size (two-bubble asymmetric mode). However, turbulent detachment on both sides occurs towards the rearward. The centroid of the eddies is quasi symmetric (see table 3) though they differ in size, the top one being smaller than its counterpart on the bottom side. This is due to the differences in the position of the separation point, which is not symmetric. Nonetheless, the saddle point is displaced only -0.0015 off the wake centreline being indistinguishable from the point $L_{r}$ in figure $7 \mathrm{~b}$.

As the Reynolds number increases and the flow enters the super-critical regime, the wake stabilises into a flow configuration consisting in a two-symmetric eddy pattern with the eddy centroid located closer to the wake centreline (see table 3). The turbulent separation of the flow occurs at $\phi_{s} \approx 148^{\circ}$ on both sides of the cylinder with almost no variations regardless of the Reynolds number.

By putting these parameters all together, in the super-critical regime the picture of a quite stable mean wake with minor differences in the average parameters can be visualised. 


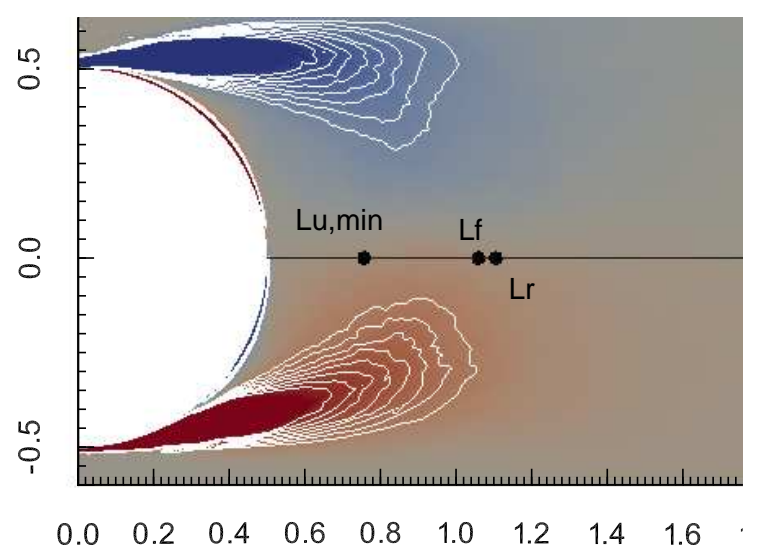

(a)

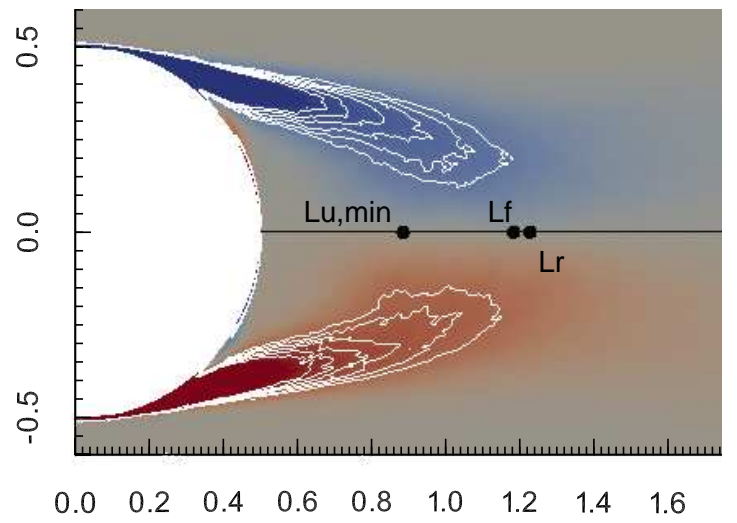

(c)

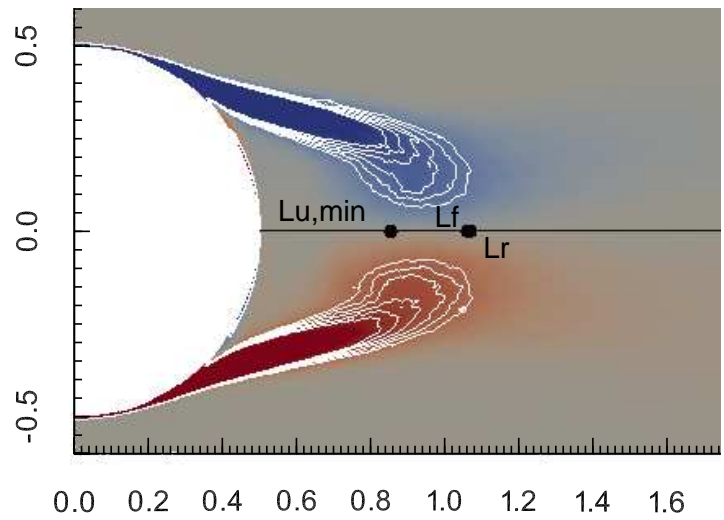

(e)

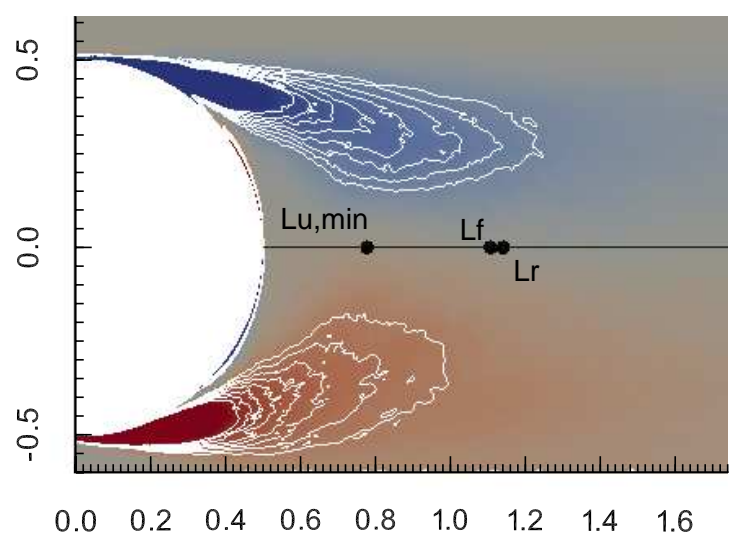

(b)

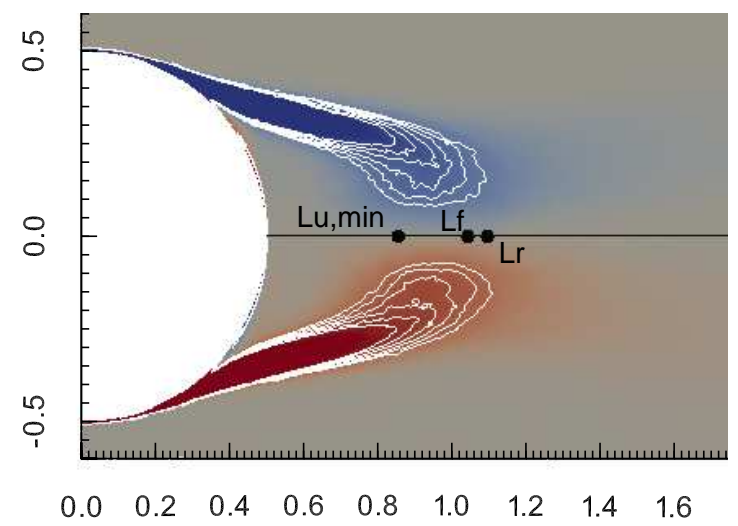

(d)

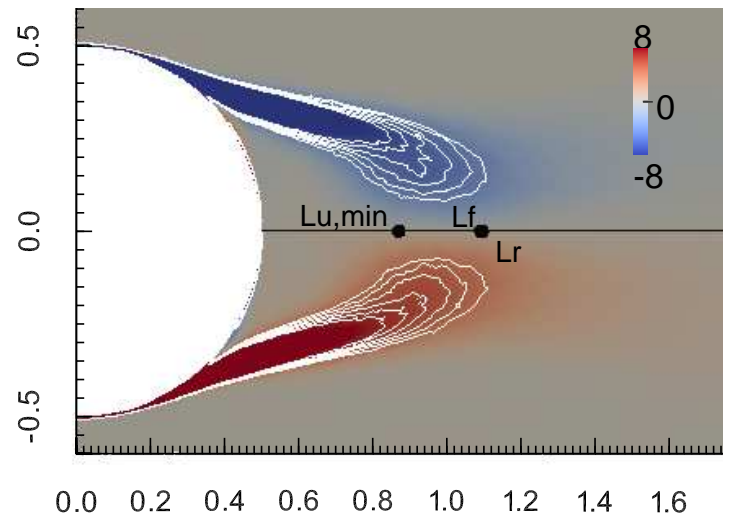

(f)

Figure 8: Contours of time-averaged non-dimensional span-wise vorticity (a) at $R e=2.5 \times$ $10^{5}$; (b) at $R e=3.8 \times 10^{5}$; (c) at $R e=5.3 \times 10^{5}$; (d) at $R e=6.5 \times 10^{5}$; (e) at $R e=7.2 \times 10^{5}$; (f) at $R e=8.5 \times 10^{5}$. In (a,b) $\bar{\omega}_{z} D / U_{\text {ref }}= \pm(2.8-7)$ and in (c-f) $\bar{\omega}_{z} D / U_{\text {ref }}= \pm(4-7)$, with $\Delta \bar{\omega}_{z} D / U_{\text {ref }}=0.6$. 


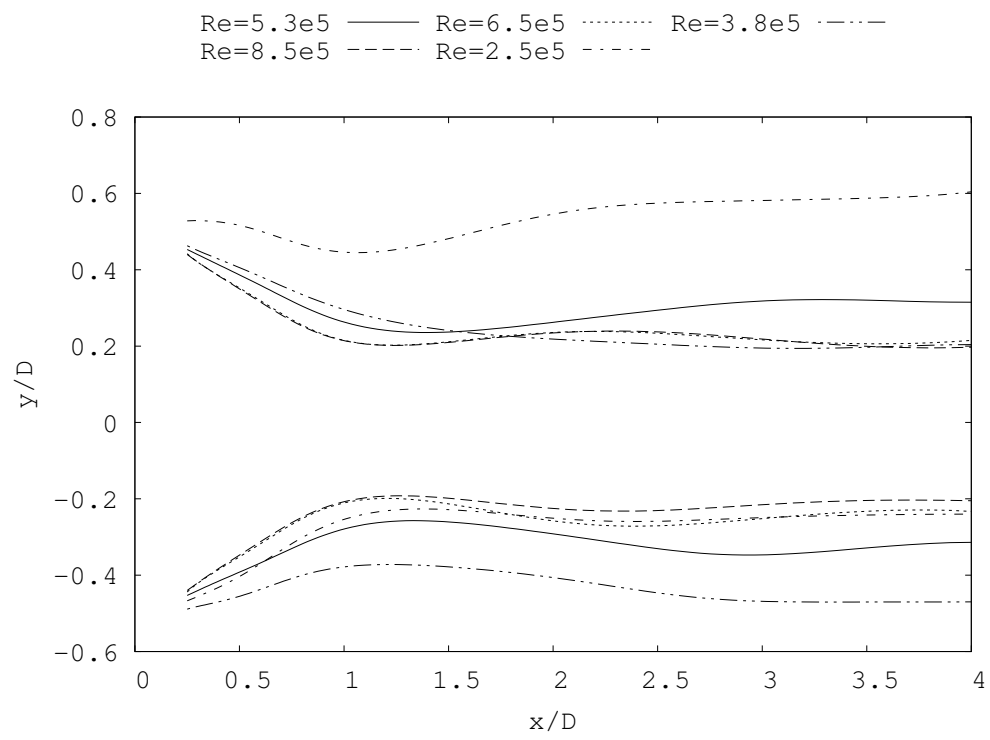

Figure 9: Variation of the local wake width at different Reynolds numbers.

In a certain way, the behaviour of the flow in this regime is somewhat similar to the subcritical one, but the transition to turbulence causes the delayed turbulent separation of the flow on the cylinder, giving rise to a more reduced recirculation region confined in the rearward of the cylinder.

The separated shear-layers are also affected by the changes in the flow and the transition to turbulence. As the flow enters the critical regime, the pressure gradient increase accelerates the flow and the shear-layers bend towards the wake centreline. This effect is more pronounced at the end of the critical regime, onset of the super-critical one, where both shear-layers come closer towards the wake centreline, where they interact with each other. This is illustrated in figure 8, where the shear-layers have been identified by means of the mean non-dimensional span-wise vorticity. Notice that vorticity is high in the shear-layers and as the shear-layers become thicker and diffuse vorticity decreases.

Another way to quantify the changes in the wake topology, especially in the critical region where the largest ones are occurring, is by measuring the wake width. The wake width is estimated, as suggested by Norberg (1986), from the positions of the maximum stream-wise velocity fluctuations at different stream-wise locations. The results for different Reynolds 
numbers are depicted in figure 9. The wake asymmetry at $R e=2.5 \times 10^{5}$ is clearly seen in the figure, with the top limit of the wake occurring at a larger vertical distance (measured from the wake centreline) than the bottom edge. As commented before, the top side of the cylinder behaves as in the sub-critical regime, thus a wider wake should be expected. However, on the bottom side, the flow is critical and with the acceleration of the flow the wake narrows. As a result, the wake width is about $d_{w} / D \approx 0.87$, which is smaller than that of the sub-critical wake $\left(d_{w} / D>1\right)$.

At $R e=3.8 \times 10^{5}$, the wake is also asymmetric but, as commented before, in the present results it is bent downward, with the bottom edge of the wake located at a larger distance from the wake centreline than the top one. As a consequence, the wake width is narrower than at $R e=2.5 \times 10^{5}$ being about $d_{w} / D \approx 0.675$. As the Reynolds number increases the wake width decreases and the symmetry in the flow is gradually recovered. When the flow enters in super-critical regime, this quantity remains almost unaffected as can be seen from the figure. Notice also that in the super-critical regime the wake width is approximately $d_{w} / D \approx 0.4$. This value is not only lower than that of the sub-critical regime $\left(d_{w} / D>1\right)$ but also almost half the value reported by Roshko (1961) for the trans-critical regime $\left(d_{w} / D \approx 0.81\right)$.

\subsection{Vortex shedding}

Unsteady lift and drag loads indicative of the fluctuating forces acting on the cylinder. Measurements of these forces in the critical and super-critical regimes are scarce (Fung, 1960; Schmidt, 1965). As unsteady lift is related to the vortex shedding, it has a periodic nature in the sub-critical regime. However, there is large controversy regarding whether vortex shedding occurs in the critical and super-critical regimes, and sometimes unsteady lift has been reported to lose coherence in these regimes (e.g. Fung, 1960; Schmidt, 1965). In section 3.1, it has been commented on the different causes of the large scattering observed in the drag coefficient measurements. However, cylinder aspect ratio and end conditions have also an important effect in the vortex shedding or the lack thereof. Achenbach and Heinecke (1981) observed that for small cylinder aspect ratios the flow behind the cylinder became three- 
dimensional and vortex shedding coherence was lost. These effects were observed for a cylinder of aspect ratio $L_{z} / D=3.38$, whereas for a model of aspect ratio $L_{z} / D=6.75$ a coherent vortex shedding was measured. This was also commented by Blackburn and Melbourne (1996), who attributed the absence of a coherent peak in the spectra in their measurements to a relative small cylinder aspect ratio of $L_{z} / D=4.5$. Furthermore, Bearman (1969) reported that in the critical and the super-critical regimes the flow is very sensitive to small changes in the experimental conditions; small disturbances on well polished surfaces, such as dust particles, affected the flow and triggered its three-dimensionality along the span, which in turn caused the regular vortex shedding ceasing.

Another possible cause of loss of coherence in the wake may be attributed to the use of a three-dimensional model in the experiments, as in Schmidt (1965) and Spitzer (1965). They both used a cantilevered cylinder of aspect ratio $L_{z} / D=8$ and, in Spitzer (1965) measurements were not made at the cylinder mid-span but at $z / D=4.86-5.87$. It has been shown both experimentally (see for instance Park and Lee, 2000) and numerically (Afgan et al., 2007) that depending on the aspect ratio of the cylinder, the flow in the near wake is rather non-uniform and that wake three-dimensionality and vortex shedding are affected by the downwash flow from the free-end of the cylinder. The latter is relevant as it was reported (for $R e=20 \times 10^{3}$ ) that as $L_{z} / D$ decreases vortex shedding frequency also decreases and spectrum bandwidth increases; vortex shedding disappears for $L_{z} / D \leq 6$. This may be the reason why the non-dimensional vortex shedding frequency detected by Spitzer (1965) at the beginning of the critical regime was of $S t=0.12$, lower than the values for a two-dimensional cylinder in the subcritical regime $S t \approx 0.2$ (see for instance Williamson, 1996), and for higher Reynolds numbers the flow seemed incoherent. The relative low aspect ratio of his model, together with the complexity of the flow in the critical regime and the fact that he did not measure at the cylinder mid-span might have contributed to the observation of a rather incoherent flow at the end of the critical regime and in the super-critical zone.

In Lehmkuhl et al. (2014), a regular von-Kármán vortex street was observed at every Reynolds numbers in the critical regime, with the exception of $R e=3.8 \times 10^{5}$ (corresponding with the two-bubble asymmetric mode according to figure 6) where the flow loses 


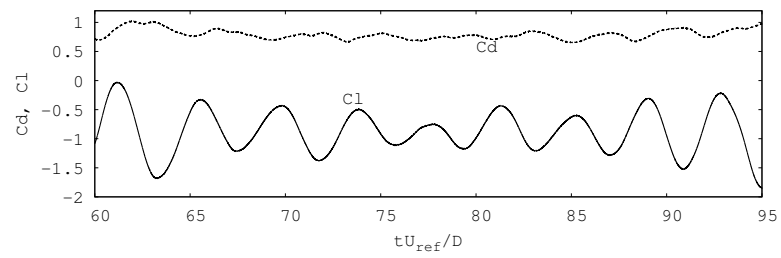

(a)

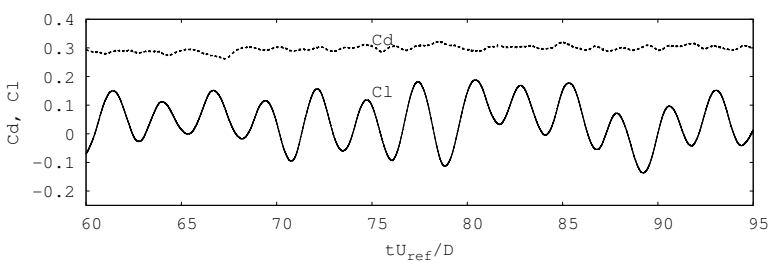

(c)

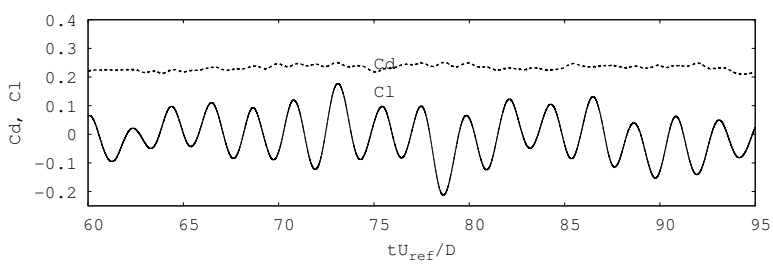

(e)

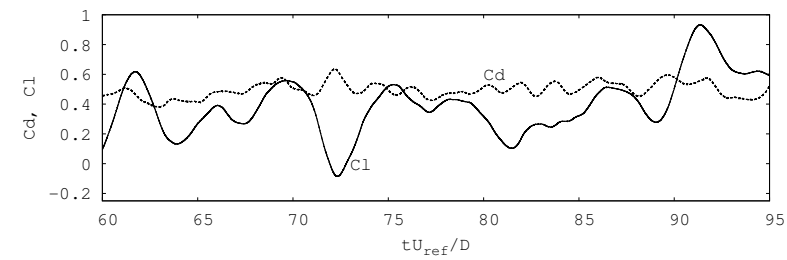

(b)

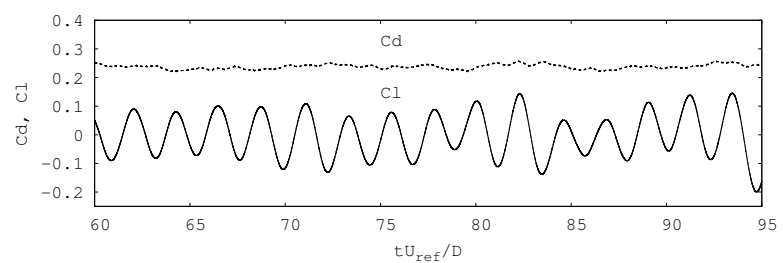

(d)

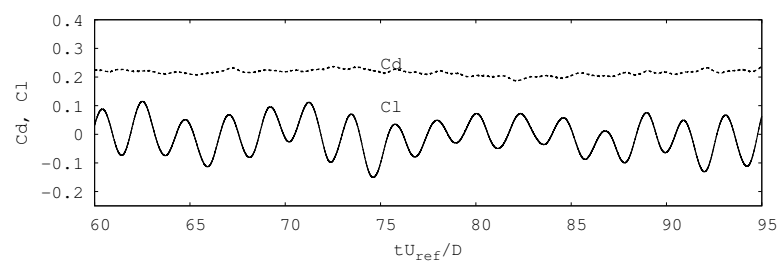

(f)

Figure 10: Instantaneous variation of the (__ l lift and ( ..........) drag coefficients (a) at $R e=2.5 \times 10^{5}$; (b) at $R e=3.8 \times 10^{5} ;(\mathrm{c})$ at $R e=5.3 \times 10^{5}$; (d) at $R e=6.5 \times 10^{5}$; (e) at $\left.R e=7.2 \times 10^{5}\right) ;(\mathrm{f})$ at $R e=8.5 \times 10^{5}$.

some coherence due to the asymmetries in the shear-layers and, instead of one peak in the frequency spectrum, two smaller were found. Moreover, non-dimensional vortex shedding frequency was found to change from $S t=0.235$ at the bottom part of the critical regime to rise up until $S t \sim 0.44$ at the end of this regime $\left(R e=6.5 \times 10^{5}\right)$.

In figure 10, the unsteady signals of the lift and drag coefficients at all Reynolds numbers studied are plotted. As can be seen, at $R e=2.5 \times 10^{5}$ (see figure 10a), the average magnitude of the steady lift is greater than zero (corresponding with the values given in Table 2) due to the asymmetry of the flow commented before. Nonetheless, the instantaneous lift exhibits a 


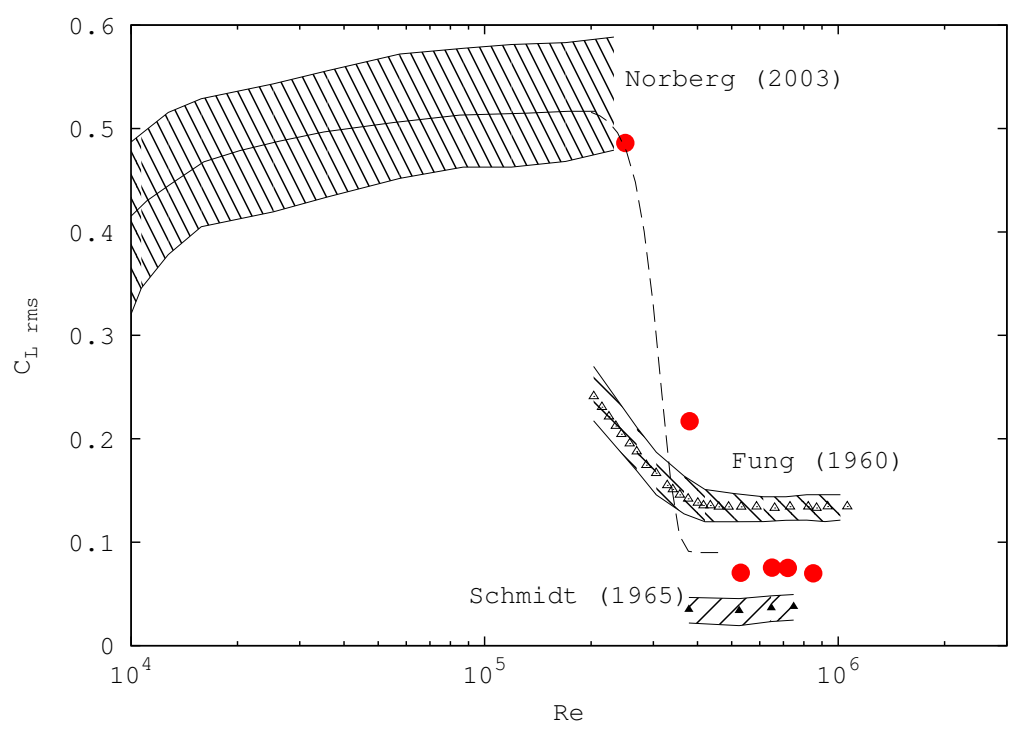

Figure 11: Fluctuating lift as a function of the Reynolds numbers. Comparison with avail-

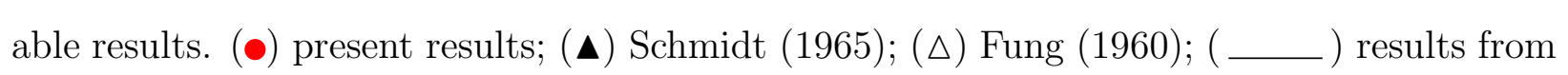
Norberg (2003); ( - - - ) empirical function $C_{L, r m s}=0.09+0.43 \exp \left[-10^{5}\left(\operatorname{Re} / 10^{6}\right)^{10}\right]$ from Norberg (2003). The dashed zone represents the bandwidth of the experimental measurements.

periodic behaviour with a large magnitude in the amplitude of its fluctuations $C_{L, r m s} \approx 0.49$. This value, which is quite close to those measured in the sub-critical regime (see also figure 11), is also correlated to the wider wake width measured.

The changes in the critical regime; i.e. the increase in the base pressure, the steep drop in the drag, amongst others; come also with an increase in the vortex shedding frequency and a decrease in the vortex strength. Notice the change in scale between figures 10a and 10c-f. This is reflected in the average magnitude of lift fluctuations shown in figure 11. In the figure, the experimental measurements by Fung (1960) and Schmidt (1965) together with those summarised by Norberg (2003) for the sub-critical regime are also plotted as reference. In general, the agreement is fair, the rapid decrease in the fluctuating lift is well captured. Current results follow the trend predicted by the correlation proposed by Norberg (2003) in the critical regime. In the super-critical regime, the fluctuating lift is 
almost constant regardless of the Reynolds number. The present results fall between the measurements by Fung (1960) and Schmidt (1965). In this regard, it should be commented that Schmidt (1965) pointed out that these experimental differences might be due to facts such as the model surface condition or end effects. Fung (1960) also reported the sensible nature of lift fluctuations which could be altered by any small geometrical change or even axial perturbations. Another issue to be here considered is the fact that both Fung (1960) and Schmidt (1965) reported that the unsteady lift loads did not show any periodic behaviour whatsoever. In the current simulations, the periodicity of the lift fluctuations can readily be seen from the plots of figure 10, with the exception of $R e=3.8 \times 10^{5}$, where the coherence in the signal is not that clear. This issue has been explained in Lehmkuhl et al. (2014), and it is due to the randomness in the interaction between both shear-layers, as they both bend towards the wake centreline but with different angles.

Figure 12 shows the power spectra of the lift fluctuations for all Reynolds numbers studied. By means of the analysis of the spectra, the coherence (or lack thereof) of lift fluctuations can be extracted. Out of $R e=3.8 \times 10^{5}$, all spectra show a distinguishable peak at the vortex shedding frequency. At $R e=3.8 \times 10^{5}$, two peaks in the spectrum are observed (see figure 12b) at $S t_{1}=0.238$ and $S t_{2}=0.358$, which agree with the values obtained by means of the power spectra of velocity fluctuations at different stations in the near wake (Lehmkuhl et al., 2014). This spectrum shows that the flow is in transition and the coherence is almost lost. Vortex strength is lower than at $R e=2.5 \times 10^{5}$, denoted by the smaller peaks obtained. There are also fluctuations of low-frequency and high intensity which is another indication of this transition in the flow.

At $R e=5.3 \times 10^{5}$, the symmetry in the flow is almost recovered and is reflected by the high coherence of the signal in the spectrum. However, non-dimensional vortex shedding frequency $(S t=0.368)$ is lower than that at super-critical Reynolds numbers due to a wider separation between both shear-layers caused by the lower base pressure and lower pressure gradient (see also figure 8c vs. figures 8d-f).

In the super-critical regime, a steady plateau is reached, with vortex shedding about $S t \approx 0.44$ at all Reynolds numbers. These observations are in good agreement with Schewe 
(a)

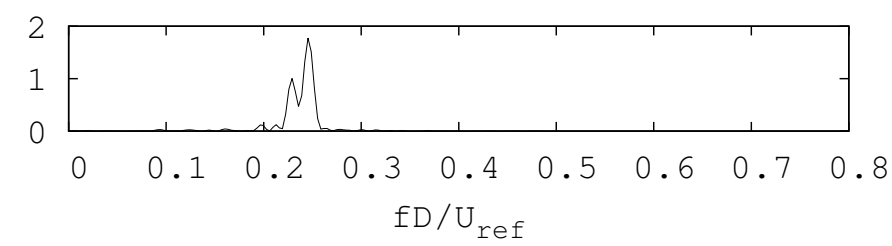

(b)

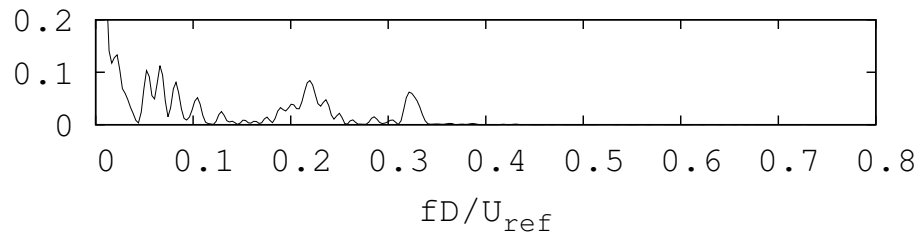

(c)

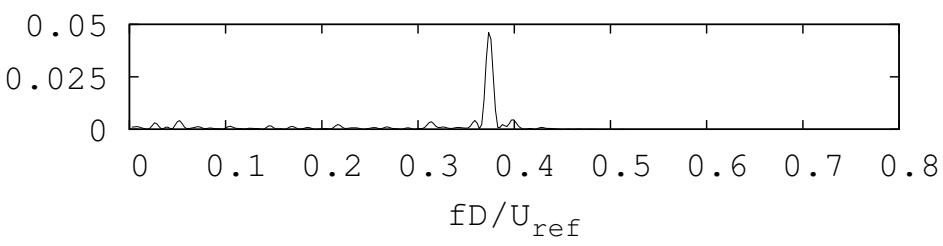

(d)

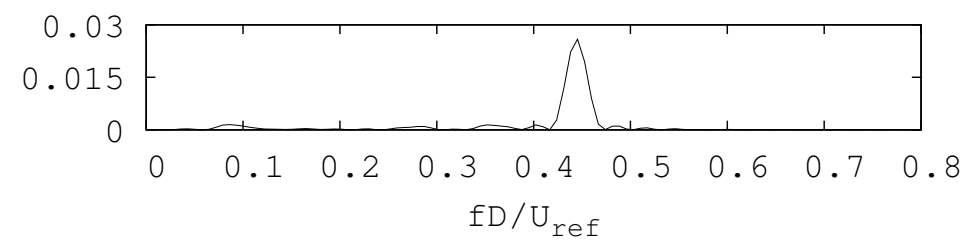

(e)

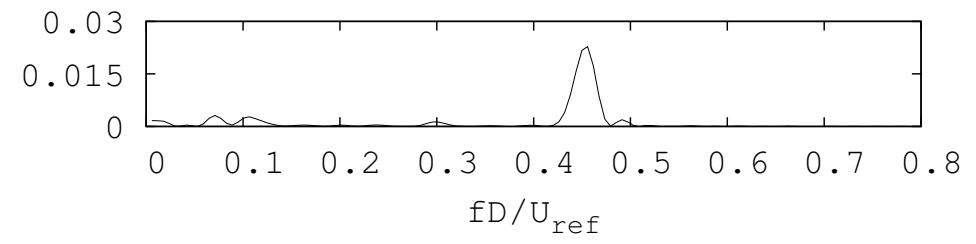

(f)

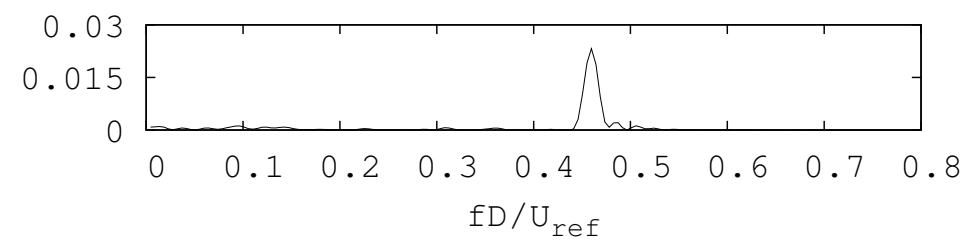

Figure 12: Power spectra of the lift fluctuations at Reynolds numbers: (a) at $R e=2.5 \times 10^{5}$; (b) at $R e=3.8 \times 10^{5}$; (c) at $R e=5.3 \times 10^{5}$; (d) at $R e=6.5 \times 10^{5}$; (e) at $R e=7.2 \times 10^{5}$ and (f) at $R e=8.5 \times 10^{5}$. 


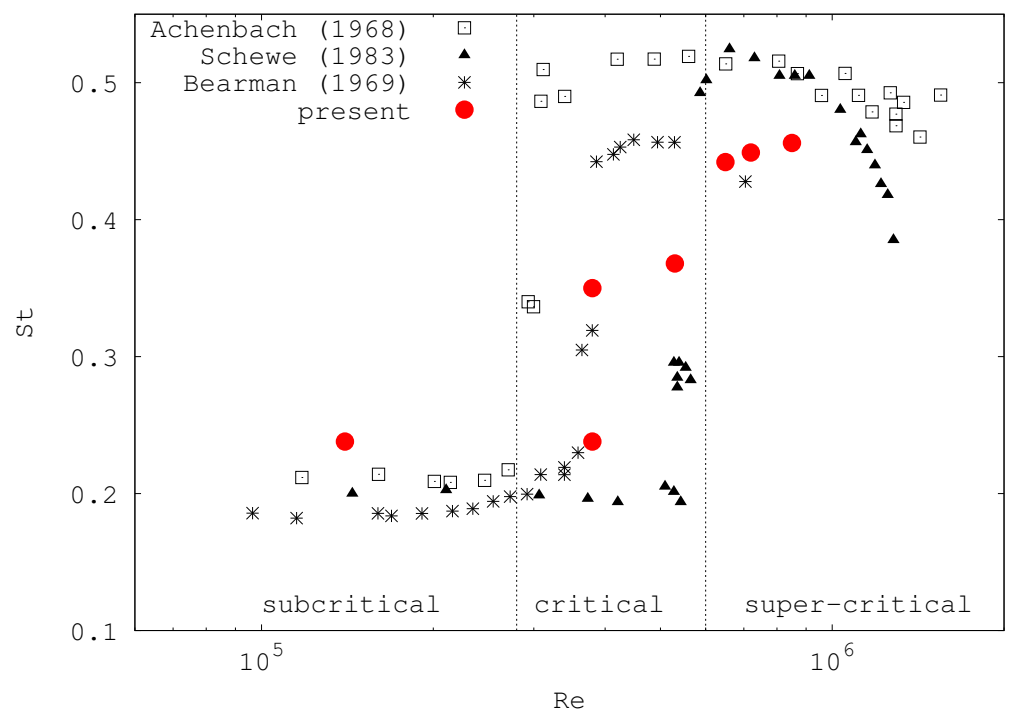

Figure 13: Non-dimensional vortex shedding frequency at critical and super-critical Reynolds numbers. Comparison with literature results. (๑) present LES; $\left(^{*}\right)$ Bearman (1969); (๑) Achenbach (1968); ( $\mathbf{\Delta}$ ) Schewe (1983).

(1983). Contrary to other experimental results (e.g. Schmidt, 1965; Jones, 1968; Shih et al., 1993) he did measure a periodic flow at super-critical Reynolds numbers. He also commented that the super-critical regime was characterised by a very stable flow with low fluctuations and a narrow band power spectrum of lift fluctuations. However, the value of lift fluctuations reported in his work was of 0.02 , which is lower than the values obtained in the present computations.

The non-dimensional vortex shedding frequency obtained is summarised in figure 13 and compared to those experimental results where vortex shedding was successfully measured. The agreement is rather good though some scatter between them is observed, mainly due to the critical nature of the flow in this range which makes more difficult the repeatability of the results.

Roshko (1954) proposed a universal relation for the wake non-dimensional vortex shedding based on the wake width $d_{w}$, and he concluded that this universal Strouhal number is $S t^{*}=0.164$ regardless of the bluff body shape. 
According to Roshko (1954),

$$
\frac{S t}{S t^{*}}=\frac{D}{d_{w}}\left(1-C_{p b}\right)^{1 / 2}
$$

Roshko (1961) used this scaling in the trans-critical regime and verified its validity, though for the super-critical regime he was unable to corroborate it. He speculated whether inaccurate measurements or the lack of coherence of the flow might be the cause why this expression would not be suitable.

Using Roshko correlation, the ratio of the non-dimensional vortex shedding frequency measured in the present numerical experiments to the non-dimensional vortex shedding obtained $\left(S t_{c o r r}\right)$ with equation $[6$ is evaluated. Results are plotted in figure 14. For the particular case of $R e=3.8 \times 10^{5}$, in which two frequencies were identified, the frequency plotted is taken as the mean value. In spite of some scatter, one can observe that this ratio is rather close to the unity for all Reynolds numbers. These observations extend Roshko's (1961) conclusions regarding the similarity of the vortex shedding phenomenon in the trans-critical regime with that of the sub-critical regime, to both critical and supercritical zones showing the continuity in the vortex shedding nature in the whole range of Reynolds numbers. It is worth noting that in equation 6 the actual value of the wake width was used, rather than obtain it from theoretical considerations as Roshko did.

Last but not the least, we would draw the attention to the onset of the instabilities in the shear-layer. It was shown in Lehmkuhl et al. (2014) that in the critical regime, as long as the attached boundary layers have not undergone turbulent, instabilities of the shear-layer upon separation can be correlated to the vortex shedding frequency, following the correlation proposed by Prasad and Williamson (1996). The same reasoning would be here applied for the super-critical regime. Figure [15] depicts the ratio of the shear-layer instabilities to the vortex shedding frequency $f_{K H} / f_{v s}$ for all Reynolds numbers. It can be seen that $f_{K H} / f_{v s}$ follows the same trend in the super-critical regime as in the critical one. Thus, it would be reasonable to suggest that this correlation might be valid for the whole super-critical regime, where there is a laminar separation bubble followed by turbulent separation, up until fully 


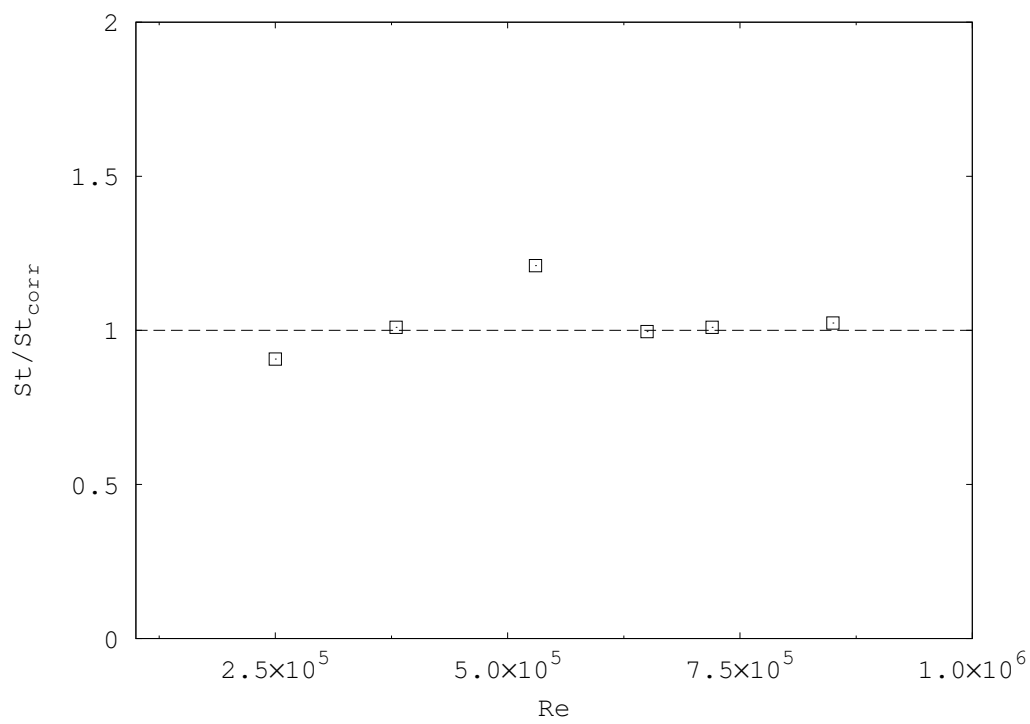

Figure 14: Ratio of the vortex shedding frequency to the vortex shedding obtained with Roshko's (1954) correlation (equation [6).

turbulent separation of the boundary layer takes place in the trans-critical regime.

\section{Conclusions}

The wake characteristics and the vortex shedding process in the flow past a circular cylinder have been studied by means of large-eddy simulations for the range of Reynolds numbers $2.5 \times 10^{5} \leq R e \leq 8.5 \times 10^{5}$, encompassing the critical and super-critical regimes. In this range, three configurations are observed: i) one-bubble asymmetric mode, with flow separation delayed on one side of the cylinder while the other remains sub-critical; ii) twobubble asymmetric mode, with a small second bubble starting to be formed and; iii) twobubble symmetric mode, with two symmetric bubbles on each side of the cylinder, which is a characteristic trait of the super-critical regime. In the first two modes (occurring at critical Reynolds numbers), the flow is asymmetric and the wake is bent towards the side with lower pressure gradient. The average flow exhibits a two-eddy pattern, but vortices formed are of different sizes and shapes. In the third mode (at super-critical Reynolds numbers), the average wake is symmetric and the eddies formed are confined to the cylinder rearward, as 


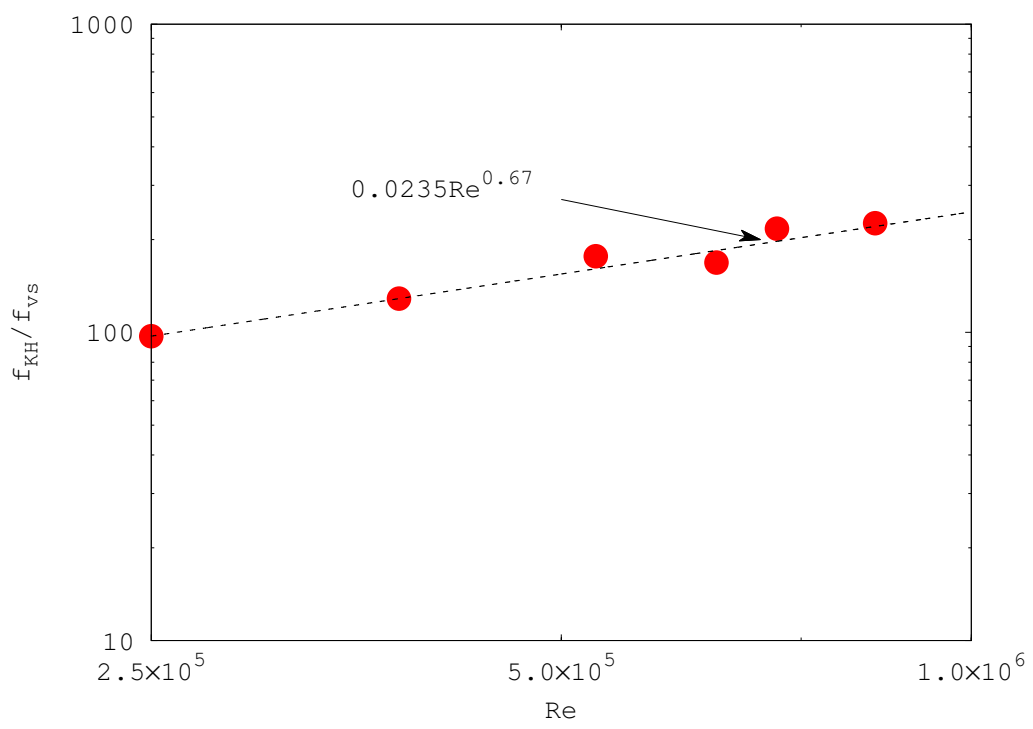

Figure 15: Ratio of the shear-layer instability to the vortex shedding frequency as a function of the Reynolds number. ( _... ) $f_{K H} / f_{v s}=0.0235 R e^{0.67}$ correlation from Prasad and Williamson (1996).

flow separation occurs at $\phi_{s} \approx 148^{\circ}$. In this mode, little changes in the wake configuration are observed.

Lift fluctuations are found to decrease from a value of $C_{L, r m s} \approx 0.49$ at the initial part of the critical regime (at $R e=2.5 \times 10^{5}$ ), close to those reported at the end of the subcritical regime (Norberg, 2003), to $C_{L, r m s} \approx 0.07$ for the super-critical Reynolds numbers (two-bubble symmetric flow). This drop in the fluctuating lift, also associated to the wake narrowing, has been found to be in rather good agreement with experimental measurements.

In spite of the major changes occurring at these Reynolds numbers, evidence of the wake vortex shedding has been obtained for all cases examined by means of the analysis of the energy spectra of the lift fluctuations. The exception might be at $R e=3.8 \times 10^{5}$ (two-bubble asymmetric mode) where coherence is considerably reduced due to the randomlike interaction of the bent shear-layers. However, coherence is rapidly recovered with the symmetric formation of the second bubble. This has been observed as a narrow band peak in the spectra, which moves towards higher frequencies as the Reynolds number increases 
in the critical regime, to then remain almost constant about $S t \approx 0.44$ throughout the super-critical regime. Furthermore, using the wake width measured as the vertical distance between maximum stream-wise fluctuations, wake vortex shedding is found to follow the universal scaling with the wake width proposed by Roshko (1954).

Following the observations made in Lehmkuhl et al. (2014), the shear-layer instability mechanism seems to remain unaltered in the super-critical regime, as transition to turbulence occurs upon separation. The ratio of the fundamental frequency of the shear-layer to the wake vortex shedding shows the same dependency with the Reynolds number as proposed

by Prasad and Williamson (1996). It would then be reasonable to suggest that this might remain valid for the whole super-critical regime as long as the attached boundary layer is still laminar.

\section{Acknowledgements}

This work has been partially financially supported by the Ministerio de Economía y Competitividad, Secretaría de Estado de Investigación, Desarrollo e Innovación, Spain (ref. ENE2009-07689) and by the collaboration project between Universitat Politècnica de Catalunya and Termo Fluids S.L. We acknowledge PRACE (project 2012071290) for awarding us access to the MareNostrum III machine based in Barcelona, Spain.

\section{AppendixA. Experimental details for measurements from the literature}

A compilation of the measurement conditions for most of the experimental data presented in this paper is given in table A.4. In the table, together with the range of Reynolds numbers at which the experiments were carried out, the spanwise size of the model $L_{z} / D$, the blockage ratio $D / L_{y}$, the roughness of the model $k_{s} / D$, the inlet turbulence level $T u$, defined as $T u=u_{r m s} / U_{r e f}$, and the Mach number of the wind tunnel $M a$ are also given. In most of these experiments the exact value of the surface roughness was not reported. Instead, references to highly polished or smooth surfaces were reported, as seen in the table. Notice that the experiments of Schmidt (1965) and Spitzer (1965) were performed with a 
cantilevered cylinder. The conditions reported in the table for Wieselsberger (1922) are for the cylinder of diameter $0.3 m$ used in the critical regime.

Achenbach, E., 1968. Distribution of local pressure and skin friction around a circular cylinder in cross-flow up to Re=5e6. J. Fluid Mech 34, 625-639.

Achenbach, E., Heinecke, E., 1981. On vortex shedding from smooth and rough cylinders in the range of Reynolds numbers 6e3 to 5e6. J. Fluid Mech 109, 239-251.

Afgan, I., Moulinec, C., Prosser, R., Laurence, D., 2007. Large eddy simulation of turbulent flow for wall mounted cantilever cylinders of aspect ratio 6 and 10. International Journal of Heat and Fluid Flow 28 (4), 561-574.

Bearman, P. W., 1965. Investigation of the flow behind a two-dimensional model with a blunt trailing edge and fitted with splitter plates. Journal of Fluid Mechanics 21, 241-255.

Bearman, P. W., 1969. On vortex shedding from a circular cylinder in the critical Reynolds number regime. J. Fluid Mech 37, 577-585.

Blackburn, H., 1994. Effect of blockage on spanwise correlation in a circular cylinder wake. Experiments in fluids 18 (1-2), 134-136.

Blackburn, H., Melbourne, W., 1996. The effect of free-stream turbulence on sectional lift forces on a circular cylinder. Journal of Fluid Mechanics 11, 267-292.

Bloor, M. S., Gerrard, J. H., 1966. Measurements on turbulent vortices in a cylinder wake. Proceedings of the Royal Society A: Mathematical, Physical and Engineering Sciences 294 (1438), 319-342.

Borrell, R., Lehmkuhl, O., Trias, F. X., Oliva, A., 2011. Parallel direct Poisson solver for discretisations with one Fourier diagonalisable direction. Computational Physics 230 (12), $4723-4741$.

Braza, M., Perrin, R., Hoarau, Y., 2006. Turbulence properties in the cylinder wake at high Reynolds numbers. Journal of Fluids and Structures 22, 757-771. 
Table A.4: Compilation of the measurement details for the experimental data presented. * The roughness is given whenever possible. $\ddagger$ Experimental measurements on a cantilevered cylinder. $†$ Experimental measurements from Vaz et al. (2007) were in a towing tank. NR means not reported.

\begin{tabular}{|c|c|c|c|c|c|c|}
\hline Reference & $R e$ & $L_{z} / D$ & $D / L_{y}[\%]$ & $k_{s} / D^{*}$ & $T u[\%]$ & $M a$ \\
\hline Wieselsberger (1922) & $4.2-8 \times 10^{5}$ & 5 & 15 & polished & NR & $<0.1$ \\
\hline Fage $(\underline{1930})$ & $10^{5}-8 \times 10^{5}$ & $8.1-16.4$ & $6-12.3$ & polished & NR & $<0.06$ \\
\hline Bursnall and Loftin (1951) & $2 \times 10^{5}-5 \times 10^{5}$ & 18 & 2.2 & smooth & $\sim 0.01$ & $<0.2$ \\
\hline Delanv and Sorensen (1953) & $10^{4}-2 \times 10^{6}$ & $6-30$ & $4.8-23.8$ & highly polished & NR & $<0.4$ \\
\hline Fung (1960) & $3 \times 10^{5}-1.4 \times 10^{6}$ & 5.7 & 10.5 & NR & $\mathrm{NR}$ & NR \\
\hline Roshko (1961) & $10^{6}-10^{7}$ & 5.66 & 13.6 & $\sim 10^{-5}$ & NR & $<0.25$ \\
\hline$\underline{\underline{\text { Schmidt }}}(\underline{1965})^{\ddagger}$ & $3.8 \times 10^{5}-7.5 \times 10^{5}$ & 8 & 7.1 & polished & 0.35 & $<0.2$ \\
\hline$\underline{\text { Spitzer }}(\underline{1965})^{\ddagger}$ & $2.5 \times 10^{5}-7.5 \times 10^{5}$ & 8 & 7.1 & polished & 0.35 & $<0.2$ \\
\hline Achenbach (1968) & $6 \times 10^{3}-5 \times 10^{6}$ & $3.3-6.6$ & 16.6 & $1.3 \times 10^{-5}$ & 0.7 & $<0.1$ \\
\hline Jones (1968) & $3.6 \times 10^{5}-18.7 \times 10^{6}$ & 5.3 & 18.8 & smooth & NR & $0.2-0.6$ \\
\hline Bearman $(\underline{1969})$ & $10^{5}-7.5 \times 10^{5}$ & 12 & 6.5 & highly polished & 0.2 & $<0.18$ \\
\hline Achenbach and Heinecke (1981) & $1.5 \times 10^{5}-4 \times 10^{6}$ & $3.38-6.75$ & 16.6 & $\sim 10^{-5}$ & 0.45 & NR \\
\hline Schewe (1983) & $2.3 \times 10^{4}-7.1 \times 10^{6}$ & 10 & 10 & polished & $<0.4$ & $<0.1$ \\
\hline 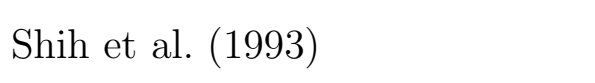 & $3.2 \times 10^{5}-6.3 \times 10^{6}$ & 8 & 11 & $2.5 \times 10^{-5}$ & $\leq 0.08$ & $<0.3$ \\
\hline Vaz et al. $(\underline{2007})^{\dagger}$ & $3 \times 10^{4}-9 \times 10^{5}$ & 18 & 5 & smooth & NR & - \\
\hline
\end{tabular}


Breuer, M., 2000. A challenging test case for large eddy simulation: high Reynolds number circular cylinder flow. International Journal of Heat and Fluid Flow 21 (5), 648-654.

Bursnall, W., Loftin, L. J., 1951. Experimental investigation of the pressure distribution about a yawed circular cylinder in the critical Reynolds number range. Tech. Rep. NACA TN2463, NACA.

Cantwell, B., Coles, D., 1983. An experimental study of entrainment and transport in the turbulent near wake of a circular cylinder. Journal of Fluid Mechanics 136, 321-374.

Catalano, P., Wang, M., Iaccarino, G., Moin, P., 2003. Numerical simulation of the flow around a circular cylinder at high Reynolds numbers. International Journal of Heat and Fluid Flow 24 (4), 463-469.

Delany, N., Sorensen, N., 1953. Low-speed drag of cylinders of various shapes. Tech. Rep. NACA TN3038, NACA.

Dryden, H., Heald, R., 1926. Investigation of turbulence in wind tunnels by a study of the flow about cylinders. Tech. Rep. 231, National Advisory Committee for Aeronautics.

Fage, A., 1930. Drag of circular cylinders and spheres. Tech. Rep. R\&M 1370, Aeronautical Research Council.

Fung, Y., 1960. Fluctuating lift and drag acting on a cylinder in a flow at supercritical Reynolds numbers. Journal of the aerospace science 27 (11), 801-814.

Humphreys, J. S., 1960. On a circular cylinder in a steady wind at transition Reynolds numbers. Journal of Fluid Mechanics 9 (4), 603-612.

Jofre, L., Lehmkuhl, O., Ventosa, J., Trias, F. X., Oliva, A., 2014. Conservation properties of unstructured finite-volume mesh schemes for the navier-stokes equations. Numerical Heat Transfer, Part B: Fundamentals 54 (1), 53-79. 
Jones, G. W., 1968. Steady lift forces generated by vortex shedding about a large, stationary, and oscillating cylinder at high Reynolds numbers. In: Fluids Engineering Conference. Vol. ASME PAPER 68-FE-36.

Karabelas, S., 2010. Large Eddy Simulation of high-Reynolds number flow past a rotating cylinder. International Journal of Heat and Fluid Flow 31 (4), 518-527.

Lehmkuhl, O., Rodríguez, I., Borrell, R., Chiva, J., Oliva, A., 2014. Unsteady forces on a circular cylinder at critical Reynolds numbers. Physics of Fluids 26 (12), 125110.

Moussaed, C., Salvetti, M. V., Wornom, S., Koobus, B., Dervieux, A., 2014. Simulation of the flow past a circular cylinder in the supercritical regime by blending RANS and variational-multiscale LES models. Journal of Fluids and Structures 47, 114-123.

Nicoud, F., Ducros, F., 1999. Subgrid-scale stress modelling based on the square of the velocity gradient tensor. Flow, Turbulence and Combustion 62, 183-200.

Norberg, C., 1986. Interaction between freestream turbulence and vortex shedding for a single tube in cross-flow. Journal of Wind Engineering and Industrial Aerodynamics 23, $501-514$.

Norberg, C., 2003. Fluctuating lift on a circular cylinder: review and new measurements. Journal of Fluids and Structures 17 (1), 57-96.

Park, C.-W., Lee, S.-J., 2000. Free end effects on the near wake flow structure behind a finite circular cylinder. Journal of Wind Engineering and Industrial Aerodynamics 88 (23), 231-246.

Polhamus, E. C., 1984. A review of some Reynolds number effects related to bodies at high angle of attack. Tech. Rep. NASA Contractor Report 3809, NASA.

Prasad, A., Williamson, C. H. K., 1996. The instability of the separated shear layer from a bluff body. Physics of Fluids 8, 1347. 
Relf, E., Simmons, L., 1924. On the frequency of the eddies generated by the motion of circular cylinders through a fluid. Reports and Memoranda 917, Aeronautical Research Council.

Rodríguez, I., Borrell, R., Lehmkuhl, O., Pérez-Segarra, C. D., Oliva, A., 2011. Direct numerical simulation of the flow over a sphere at $\mathrm{Re}=3700$. Journal of Fluid Mechanics $679,263-287$.

Roshko, A., 1954. On the drag and shedding frequency of two-dimensional bluff bodies. Tech. Rep. TN3169, National Advisory Committee for Aeronautics.

Roshko, A., 1961. Experiments on the flow past a circular cylinder at very high Reynolds number. Journal of Fluid Mechanics 10 (3), 345-356.

Schewe, G., 1983. On the force fluctuations acting on a circular cylinder in crossflow from subcritical up to transcritical Reynolds numbers. Journal of Fluid Mechanics 133, 265-285.

Schmidt, L. V., 1965. Measurements of fluctuating air loads on a circular cylinder. Journal of Aircraft 2 (1), 49-55.

Shih, W., Wang, C., Coles, D., Roshko, A., 1993. Experiments on flow past rough circular cylinders at large Reynolds numbers. Journal of Wind Engineering and Industrial Aerodynamics 49, 351-368.

Spitzer, R., 1965. Measurements of unsteady pressures and wake fluctuations for flow over a cylinder at supercritical Reynolds number. Ph.D. thesis, California Institute of Technology.

Szechenyi, E., 1975. Supercritical Reynolds number simulation for two-dimensional flow over circular cylinders. Journal of Fluid Mechanics 70, 529-542.

Trias, F. X., Lehmkuhl, O., 2011. A self-adaptive strategy for the time integration of NavierStokes equations. Numerical Heat Transfer. Part B 60 (2), 116-134. 
Trias, F. X., Lehmkuhl, O., Oliva, A., Pérez-Segarra, C. D., Verstappen, R. W. C. P., 2014. Symmetry-preserving discretization of Navier-Stokes equations on collocated unstructured grids. Journal of Computational Physics 258, 246-267.

Van Nunen, J., 1974. Pressure and forces on a circular cylinder in a cross flow at high Reynolds numbers. In: Springer-Verlag (Ed.), Flow Induced Structural Vibrations. Berlin, pp. $748-754$.

Vaz, G., Mabilat, C., van der Wal, R., Gallagher, P., 2007. Viscous flow computations on smooth cylinders: A detailed numerical study with validation. In: 26th International Conference on Offshore Mechanics and Artic Engineering. OMAE2007. San Diego, California.

Verstappen, R. W. C. P., Veldman, A. E. P., 2003. Symmetry-preserving discretization of turbulent flow. Journal of Computational Physics 187, 343-368.

West, G., Apelt, C., 1981. Blockage and aspect ratio effects on flow past a circular cylinder for $10^{4}<R e<10^{5}$. Tech. Rep. No. CE 29, University of Queensland.

Wieselsberger, C., 1922. New data on the laws of fluid resistance. Tech. Rep. TN-84, NACA.

Williamson, C. H. K., 1996. Vortex dynamics in the cylinder wake. Annual Review of Fluid Mechanics 28 (1), 477-539.

Zan, S., 2008. Experiments on circular cylinders in crossflow at Reynolds numbers up to 7 million. Journal of Wind Engineering and Industrial Aerodynamics 96 (6-7), 880-886. 